\title{
ORIGINAL ARTICLE Oncogenic MCT-1 activation promotes YY1-EGFR-MnSOD signaling and tumor progression
}

\author{
H-Y Tseng ${ }^{1}$, Y-A Chen ${ }^{1}$, J Jen ${ }^{2}$, P-C Shen ${ }^{1}$, L-M Chen ${ }^{1}$, T-D Lin ${ }^{1}$, Y-C Wang ${ }^{2}$ and H-L Hsu ${ }^{1}$
}

Tumor cells often produce high levels of reactive oxygen species (ROS) and display an increased ROS scavenging system. However, the molecular mechanism that balances antioxidative and oxidative stress in cancer cells is unclear. Here, we determined that oncogenic multiple copies in T-cell malignancy 1 (MCT-1) activity promotes the generation of intracellular ROS and mitochondrial superoxide. Overexpression of MCT-1 suppresses p53 accumulation but elevates the manganese-dependent superoxide dismutase (MnSOD) level via the YY1-EGFR signaling cascade, which protects cells against oxidative damage. Conversely, restricting ROS generation and/or targeting YY1 in lung cancer cells effectively inhibits the EGFR-MnSOD signaling pathway and cell invasiveness induced by MCT-1. Significantly, MCT-1 overexpression in lung cancer cells promotes tumor progression, necrosis and angiogenesis, and increases the number of tumor-promoting $\mathrm{M} 2$ macrophages and cancer-associated fibroblasts in the microenvironment. Clinical evidence further confirms that high expression of MCT-1 is associated with an increase in YY1, EGFR and MnSOD expression, accompanied by tumor recurrence, poor overall survival and EGFR mutation status in patients with lung cancers. Together, these data indicate that the MCT-1 oncogenic pathway is implicated in oxidative metabolism and lung carcinogenesis.

Oncogenesis (2017) 6, e313; doi:10.1038/oncsis.2017.13; published online 10 April 2017

\section{INTRODUCTION}

Reactive oxygen species (ROS) generated by cell metabolism can function either as signaling molecules or as cellular toxicants. As a double-edged sword, ROS influence signaling pathways to result in beneficial or detrimental outcomes in cancer therapy. ${ }^{1-3}$ Cellular ROS levels are balanced by scavenging systems such as superoxide dismutases (SODs), peroxiredoxins and glutathione peroxidas. Excessive ROS can damage proteins, lipids and DNA, leading to cell transformation and genetic mutations that contribute to carcinogenesis.

Cancer cells are capable of escaping oxidative stress by inducing antioxidant enzymes and molecules. ROS can trigger metabolic reprogramming of cancer cells to increase tumor aggressiveness and chemoresistance. ${ }^{4,5}$ ROS released from cancer cells into the microenvironment can induce stromal oxidative stress and activate nuclear factor erythroid-2-related factor, hypoxia-inducible factor 1alpha, vascular endothelial growth factor and nuclear factor kappalight-chain-enhancer of activated B cells, which promote tumor immunity, inflammation and angiogenesis. ${ }^{6-8}$

p53 has a controversial role in ROS formation. ${ }^{9}$ ROS trigger p53 activation to regulate target genes and mediate p53-dependent apoptosis. ${ }^{10}$ Reciprocally, p53 controls cellular ROS generation by decreasing manganese superoxide dismutase (MnSOD) levels. ${ }^{11,12}$ However, p53 also upregulates MnSOD and glutathione peroxidase, leading to an imbalance in antioxidant enzymes and an increase in oxidative stress. ${ }^{13}$ Aberrant induction of MnSOD sustains mitochondrial ROS generation and AMP-activated kinase signaling that promotes tumor progression toward an aggressive stage and causes therapeutic resistance and anti-apoptotic effects. $^{14-16}$ Furthermore, overexpression of MnSOD promotes epithelial-mesenchymal transition in breast carcinoma, ${ }^{17}$ mediates tumor metastasis in nasopharyngeal carcinoma ${ }^{18}$ and elevates mitochondrial superoxide levels to activate PI3K/AKT and MMP2 in invasive pathways. ${ }^{19,20}$

Multiple copies in T-cell malignancy 1 (MCT-1) is involved in posttranscriptional regulation and translation initiation. ${ }^{21-24}$ We have previously shown that enhanced MCT-1 activity decreases the promoter function, protein stability and activity of p53; therefore, overexpression of MCT-1 advances tumorigenicity in a p53-null background. ${ }^{25-27}$ Multiple MCT-1 functions have been discovered that induce cell transformation and survival, ${ }^{28,29}$ cause catastrophic mitosis $^{25,30,31}$ and promote genomic instability. ${ }^{25,26}$ In the MCT-1 oncogenic pathway, we have demonstrated that the expression of Shc (Src homology 2 domain-containing transforming protein) proteins is increased, which stimulates extracellular-regulated kinase and Ras signaling. ${ }^{27,29}$ When epidermal growth factor receptor (EGFR) phosphorylates Shc, Shc forms a complex with Grb2-Sos to activate AKT, extracellular-regulated kinase, Ras and JNK pathways. ${ }^{32-34}$ In this study, we aimed to investigate whether the oncogenic activity of MCT-1 augments the EGFR signaling cascade and promotes ROS generation. We identified a novel carcinoma metabolism pathway involving the MCT-1-YY1-EGFR-MnSOD network, which confers oxidative resistance to oncogenic cells, accompanied by an enhancement in the tumor microenvironment and tumor progression.

\section{RESULTS}

Overexpression of MCT-1 induces EGFR expression but inhibits p53 expression via YY1

Yin Yang 1 (YY1) is a ubiquitous transcription factor overexpressed in many types of cancers and is associated with poor

\footnotetext{
${ }^{1}$ Institute of Molecular and Genomic Medicine, National Health Research Institutes, Zhunan, Miaoli County, Taiwan and ${ }^{2}$ Department of Pharmacology, National Cheng Kung University, Tainan, Taiwan. Correspondence: Dr H-L Hsu, Institute of Molecular and Genomic Medicine, National Health Research Institutes, 35 Keyan Road, Zhunan, Miaoli County 35053, Taiwan 
prognoses. ${ }^{35-37}$ YY1 regulates EGFR and p53 gene expression. ${ }^{38,39}$ To study the oncogenic effect of MCT-1 in a wild-type p53 background, normal breast epithelial MCF-10A cells and invasive lung cancer A549 cells were transfected with a V5-tagged MCT-1 expression construct or the vehicle (control). We found that MCT-1 overexpression induced YY1 and EGFR but reduced p53 expression compared with control A549 cells (Figure 1a). Because of the increased amount of total EGFR, EGFR phosphorylation (Tyr1068 and Tyr1173) levels were also increased by MCT-1. However, the elevated levels of YY1 and EGFR were significantly reduced after targeting the MCT-1 gene (shMCT-1; clone \#3-9 and \#3-10) in A549 cells with a short hairpin RNA (shRNA) compared with cells containing scrambled shRNA (Figure 1b). Similarly, YY1 and EGFR were repressed but $\mathrm{p} 53$ was accumulated in the MCF-10A cells after MCT-1 depletion (shMCT-1, clone \#1 and \#2; Figure 1c). Consistent with the enrichment in YY1 protein induced by MCT-1 overexpression, the YY1 messenger RNA (mRNA) level also exhibited a 2.56-fold increase over the control cells $(P<0.001$; Figure 1d). Conversely, knockdown of MCT-1 in A549 cells (clones \#3-9 and \#3-10) repressed EGFR mRNA expression (Figure 1e). Actinomycin $D$ was then used to inhibit transcription and to examine the steady-state of YY1 mRNA levels (Figure 1f). The results showed that YY1 mRNA had a longer half-life $\left(T_{1 / 2}=9.5 \mathrm{~h}\right)$ in the MCT-1-overexpressing cells than in control A549 cells
$\left(T_{1 / 2}=6.5 \mathrm{~h}\right)$, indicating that MCT-1 induced $Y Y 1$, at least in part, through stabilization of YY1 mRNA.

To inspect the role of YY1 in the regulation of EGFR and p53, the YY1 gene was knocked down using an shRNA (shYY1) to suppress YY1 expression, which resulted in EGFR reduction and p53 accumulation in the MCT-1-overexpressing cells (Figure 2a, lane 4). To further study whether YY1 mediates EGFR gene activation in the MCT-1 pathway, the EGFR promoter segment $(-1102$ to -12$)$ was cloned into a pGL3 promoter-less vector. The data indicated that EGFR promoter activity in MCT-1-overexpressing cells was increased 2.54-fold over that in the control cells $(P<0.001$; Figure $2 \mathrm{~b})$, but it was repressed by $Y Y 1$ knockdown (0.84-fold, $P<0.001)$. Likewise, the EGFR mRNA level promoted by MCT-1 (3.73-fold, $P<0.001$ ) was inhibited after $Y Y 1$ reduction $(0.93$-fold, $P<0.001$ ) compared with control cells (Figure $2 \mathrm{c}$ ). Furthermore, the p53 promoter segment $(-188$ to +23$)$ was cloned into a pGL3 basic vector, and the promoter activity was significantly inhibited upon MCT-1 induction (0.38-fold, $P<0.001)$ but induced after YY1 knockdown $(0.97$-fold, $P<0.001)$ compared with scrambled knockdown (Figure 2d). These results confirm that $Y Y$ activates EGFR gene expression but inhibits p53 gene expression in the MCT-1 pathway.

To determine whether p53 regulates YY1 and EGFR in the MCT-1 pathway, the expression of p53 mRNA was abrogated with shRNA in MCF-10A cells (Supplementary Figure 1a). The EGFR a

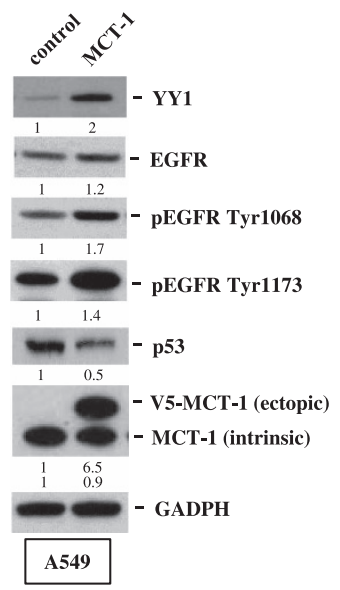

b

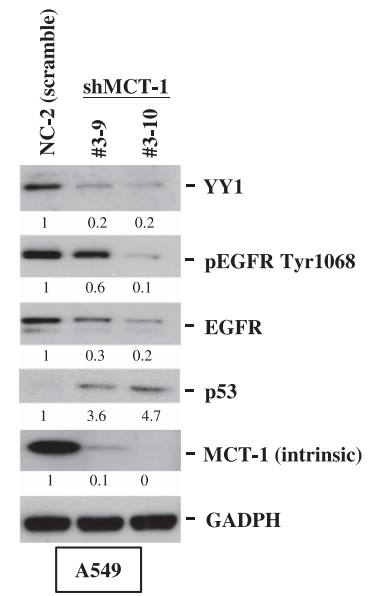

C

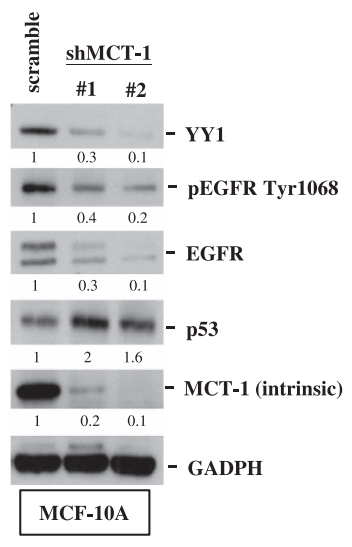

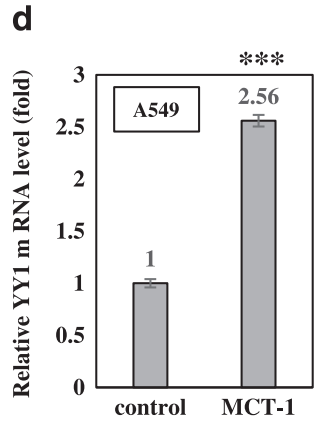
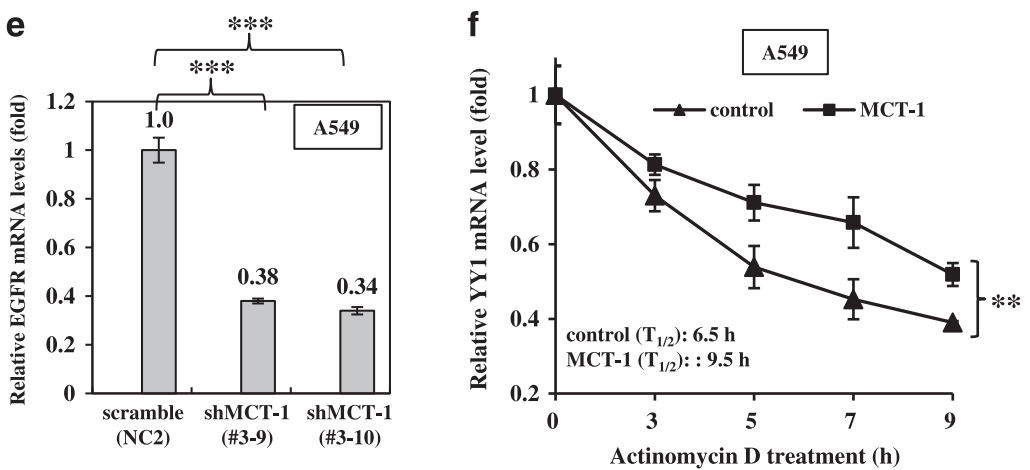

Figure 1. Overexpression of MCT-1 promotes YY1-EGFR signaling. MCF-10A cells and A549 cells overexpressing V5-tagged MCT-1 (V5-MCT-1) or with depleted endogenous MCT-1 (shMCT-1) and their comparative controls were analyzed. (a) The expression of YY1, EGFR and p53 was evaluated when A549 cells were starved for $24 \mathrm{~h}$ and then stimulated by EGF $(20 \mathrm{ng} / \mathrm{ml})$ and insulin $(10 \mu \mathrm{g} / \mathrm{ml})$ for $1 \mathrm{~h}$. (b) Two different clones (\#3-9 and \#3-10) of MCT-1 knockdown and scrambled knockdown (NC2) in A549 cells were analyzed for YY1, EGFR and p53 expression upon EGF/insulin stimulation for $1 \mathrm{~h}$. (c) MCF-10A cells with different degrees of MCT-1 knockdown (clone \#1 and \#2) and scrambled knockdown were examined after starvation for $24 \mathrm{~h}$ followed by EGF/insulin stimulation for $1 \mathrm{~h}$. The protein amounts were normalized to GAPDH, and the phosphorylated EGFR levels were normalized to total EGFR before comparison with the comparative controls. (d) YY1 mRNA levels were surveyed in the A549 cells with different MCT-1 levels. (e) Relative EGFR mRNA levels were quantified in different A549 lines with MCT-1 knockdown (\#3-9 and \#3-10) and in A549 cells with scrambled knockdown vector (NC2). (f) The half-life ( $\left.T_{1 / 2}\right)$ of YY1 mRNA was examined in A549 cells after actinomycin D treatment. The data represent the mean \pm s.d. $(n=3)$. ${ }^{* *} P<0.01$ and ${ }^{* * *} P<0.001$. 
a
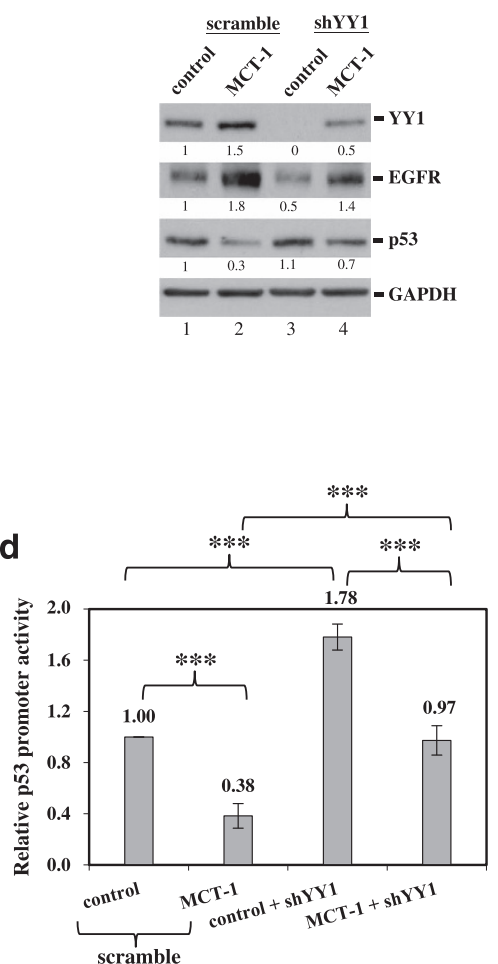

b

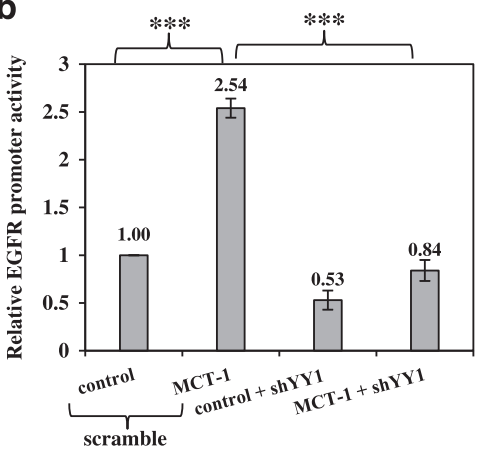

C

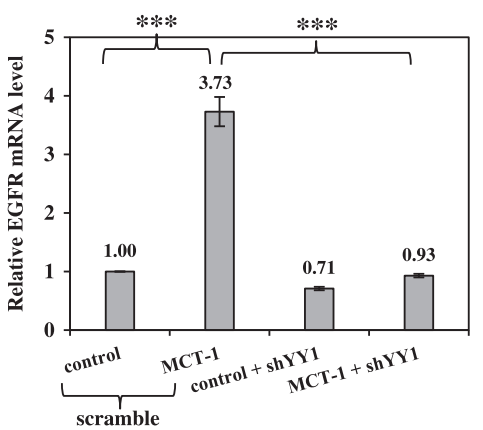

e
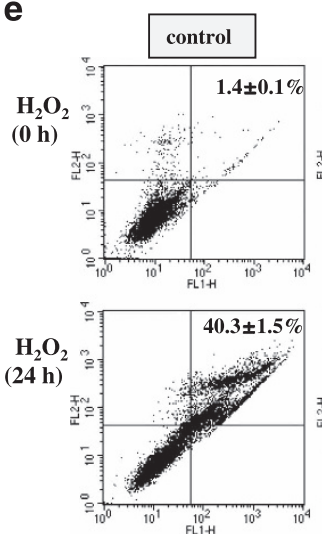
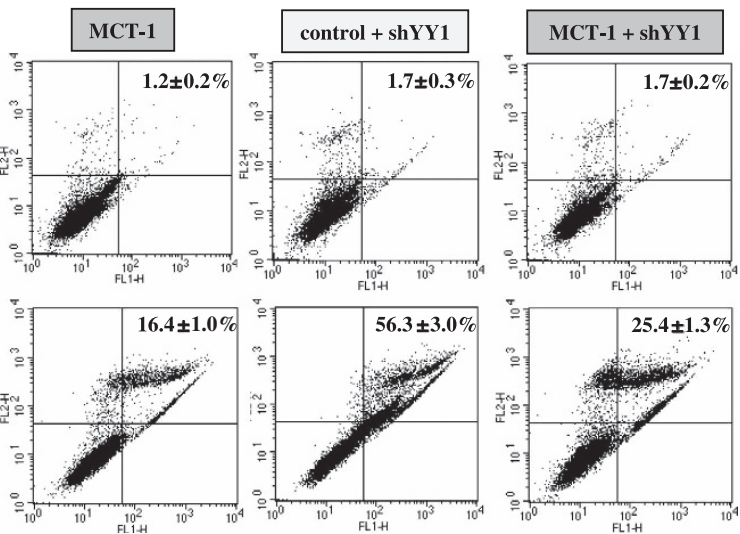

Figure 2. MCT-1 overexpression protects cells against oxidative stress via YY1. MCF-10A cells (control and MCT-1) with YY1 knockdown (shYY1) or scrambled knockdown were studied. (a) EGFR and p53 expression were analyzed in cells with different MCT-1 and YY1 levels. The protein amounts were normalized to GAPDH and compared with scrambled control (lane 1). (b) Relative EGFR promoter activity is indicated. (c) Relative EGFR mRNA level is shown. (d) Relative p53 promoter activity is shown. (e) After the cells were exposed to $100 \mu \mathrm{m} \mathrm{H}_{2} \mathrm{O}_{2}$ for $24 \mathrm{~h}$, apoptotic events were evaluated using FITC-Annexin V staining and propidium iodide (PI) counter staining, followed by flow cytometry analysis. The data represent the mean \pm s.d. $(n=3) .{ }^{* * *} p<0.001$.

promoter activity (Supplementary Figure 1b), the EGFR mRNA level (Supplementary Figure 1c) and the expression of EGFR and YY1 protein (Supplementary Figure 1d, lane 4) were additively enhanced as p53 was depleted from the MCT-1-overexpressing cells (MCT-1/-p53) compared with the p53-deficient control cells (control/-p53). These results indicate that YY1 and p53 antagonize each other in the MCT-1 pathway.

MCT-1 cooperates with YY1 and EGFR to protect cells against oxidative stress

$\mathrm{H}_{2} \mathrm{O}_{2}$ exposure directly increases intracellular ROS levels. ${ }^{40}$ To determine whether $\mathrm{YY} 1$ is important for protecting cells against oxidative stress, MCF-10A cells were exposed to $\mathrm{H}_{2} \mathrm{O}_{2}$ for $24 \mathrm{~h}$. The data showed that the MCT-1-overexpressing cells were more refractory to oxidative cell death $(16.4 \pm 1.0 \%)$ than the control cells $(40.3 \pm 1.5 \%$; Figure 2e), as detected by fluorescein isothiocyanate (FITC)-Annexin $\mathrm{V}$ staining and flow cytometry analysis to assess apoptosis levels. Clearly, YY1 functionally protected the cells against oxidative damage because the number of apoptotic events increased as YY1 was depleted from the control cells $(56.3 \pm 3.0 \%)$ and the MCT-1-overexpressing cells (25.4 $\pm 1.3 \%)$. Similarly, the MCT-1-induced A549 cell survival was also suppressed after targeting YY1 (shYY1), as evidenced by the increased apoptotic DNA fragmentation observed in the TUNEL assay (Supplementary Figure 2a).

Upon further examination of the MCF-10A cellular response to oxidative stress (Figure $3 \mathrm{a}$ ), the activating phosphorylation of EGFR (Tyr 1068) was additively promoted by $\mathrm{H}_{2} \mathrm{O}_{2}$ exposure and MCT-1 overexpression, whereas the activating phosphorylation of p53 (Ser15) and the DNA damage marker $\mathrm{Y}-\mathrm{H} 2 \mathrm{AX}$ (Ser139) were reduced when MCT-1 was overexpressed (lane 4) compared with the control group (lane 3). A reduced DNA damage response was also observed in the MCT-1 oncogenic cells when exposed to $\mathrm{H}_{2} \mathrm{O}_{2}$ and when AG1478 inactivated EGFR phosphorylation (Tyr1068) (Supplementary Figure 2b), as indicated by the decrease in $\mathrm{Y}-\mathrm{H} 2 \mathrm{AX}$. Therefore, MCT-1 overexpression protects cells against oxidative DNA damage.

To inspect whether EGFR and MCT-1 work together against oxidative stress, wild-type (wt) EGFR was introduced to promote the oncogenic effect in MCF-10A cells (Supplementary Figure 2c). As evaluated by FITC-Annexin $\mathrm{V}$ staining and observation of apoptotic events (Figure $3 b$ ), $\mathrm{H}_{2} \mathrm{O}_{2}$-induced apoptotic effects were less frequent in the MCT-1-expressing group (14.3 $\pm 1 \%)$ than in the control cohort $(32 \pm 1.5 \%)$. However, when EGFR was overexpressed (+EGFR), oxidative cell death was relatively decreased in MCT-1-overexpressing cells $(9.8 \pm 0.6 \%)$ and significantly inhibited in control cells $(11.7 \pm 1.1 \%)$. Conversely, when AG1478 inactivated EGFR (+AG1478), $\mathrm{H}_{2} \mathrm{O}_{2}$-induced apoptotic events were dramatically promoted in control cells $(70.2 \pm 1.2 \%)$ but not in MCT-1-overexpressing cells $(16.4 \pm 0.6 \%)$. Even after treatment with AG1478, the cells co-induced with MCT-1 and EGFR were still more refractory to $\mathrm{H}_{2} \mathrm{O}_{2}$ (MCT-1+EGFR, $11.7 \pm 0.8 \%$ ) than the comparative control (control+EGFR, $30.2 \pm 1.1 \%)$. Hence, EGFR and MCT-1 co-operatively protect cells against oxidative injury.

\section{MCT-1 promotes intracellular ROS generation}

EGFR-activating mutations and oxidative stress morphologically transform MCF-10A cells and enhance their oncogenic properties. $^{37,41}$ EGFR overexpression and an EGFR-activating 
b

a

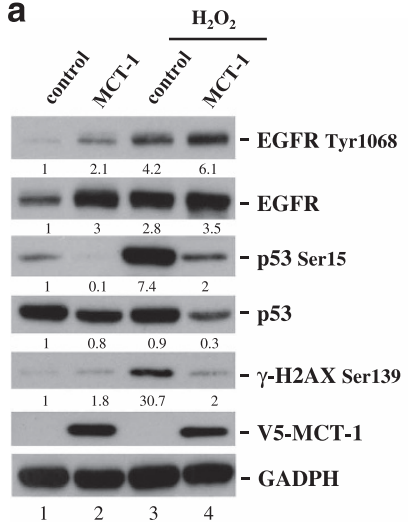

C

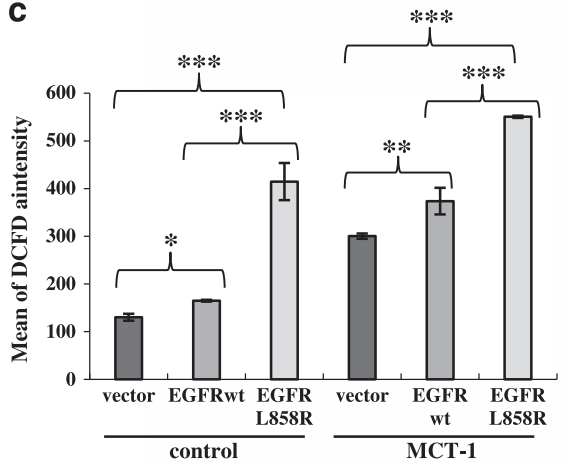

d
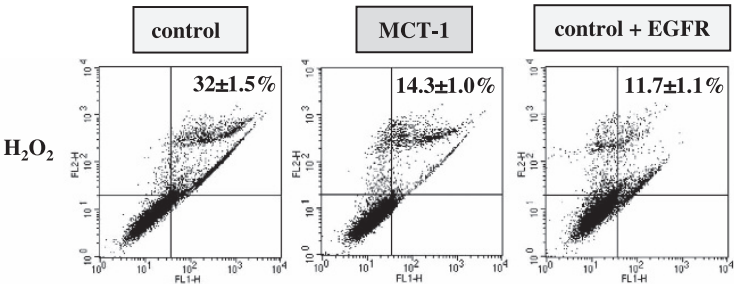

MCT-1+EGFR
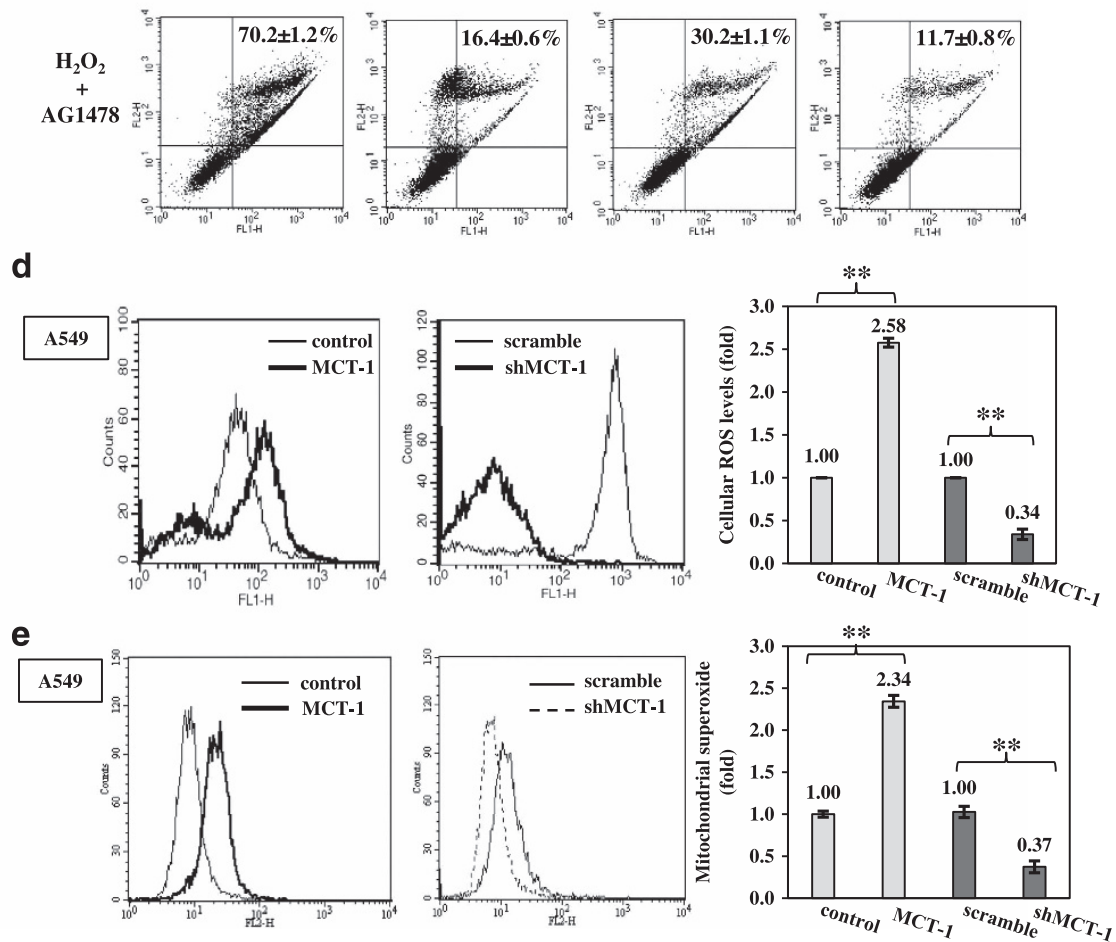

Figure 3. MCT-1 and EGFR co-operatively protect cells against oxidative stress. MCF-10A (a-c) and A549 cells (d, e) with different levels of MCT-1 expression were studied. (a) The expression and phosphorylation levels of EGFR, p53 and $\mathrm{H} 2 \mathrm{AX}$ were studied after $\mathrm{H}_{2} \mathrm{O}_{2}$ exposure for $1 \mathrm{~h}$. The protein amounts were normalized to GAPDH, and the phosphorylated EGFR levels were normalized to total EGFR before comparison with the comparative control (lane 1). (b) The cells (control, MCT-1) with or without EGFR co-expression were exposed to $\mathrm{H}_{2} \mathrm{O}_{2}$ for $24 \mathrm{~h}$ or pretreated with $5 \mu \mathrm{M}$ AG1478 for $1 \mathrm{~h}$. Flow cytometry was used to analyze the oxidative cell death after FITC-Annexin $\mathrm{V}$ staining and propidium iodide (PI) counter staining. (c) ROS generation in the cells overexpressing wild-type EGFR (EGFRwt) or mutant EGFR (L858R) was detected by a DCFDA-cellular ROS method. (d) Intracellular ROS levels were analyzed and compared in different cellular contents (MCT-1 overexpressing vs vector control and MCT-1 silencing (shMCT-1) vs. scrambled knockdown). (e) Mitochondrial superoxide levels were examined and quantified in different MCT-1 contents using mitochondrial superoxide indicator (MitoSOX) and flow cytometry analysis. Data represent the mean \pm s.d. $(n=3) .{ }^{*} P<0.05 ;{ }^{*} P<0.01 ;{ }^{* *} P<0.001$.

mutation (L858R) have been observed in triple-negative breast cancer (TNBC) ${ }^{42}$ EGFRwt and the L858R mutant were introduced into MCF-10A cells to study whether EGFRwt or the L858R mutant affect ROS generation in normal breast epithelial cells (Figure 3c). Using a DCFDA-cellular ROS detection system, we found that the intracellular ROS levels were enhanced when EGFRwt was co-induced with MCT-1, and ROS levels were further advanced when MCT-1 oncogenic cells expressed the EGFR L858R mutant. This result suggests that MCT-1 overexpression alongside EGFR overexpression or expression of mutated EGFR may develop a ROS scavenging system to adapt to an oxidative environment and maintain oncogenic cell survival.

Cancer cell metabolism continually produces superoxide $\left(\mathrm{O}_{2}^{-}\right)$ and hydrogen peroxide $\left(\mathrm{H}_{2} \mathrm{O}_{2}\right) \cdot{ }^{43}$ Upon further examination of the intrinsic ROS production in A549 cancer cells, we noted a 2.58-fold increase in the intracellular ROS level in MCT-1-overexpressing cells over the control group $(P<0.01$; Figure $3 \mathrm{~d})$. In contrast, ROS levels exhibited a 2.94-fold reduction after MCT-1 knockdown (shMCT-1) compared with scrambled knockdown $(P<0.01)$. Mitochondria are major sources of ROS generation in cancer cells. ${ }^{1}$ Using mitochondrial superoxide indicator (MitoSOX) Red to detect the superoxide level in mitochondria, a 2.34-fold elevation in mitochondrial superoxide levels was identified in MCT-1overexpressing cells (Figure 3e), whereas mitochondrial ROS showed a 2.7-fold reduction after MCT-1 knockdown. Therefore, MCT-1 oncogenic stress stimulates the formation of intracellular and mitochondrial ROS. Loss of MCT-1 effectively alleviates ROS production, presumably by improving an antioxidant mechanism.

\section{MCT-1 induces MnSOD via YY1-EGFR signaling}

Mitochondrial superoxide generation stimulates MnSOD expression in cancer cells. ${ }^{44}$ We investigated MnSOD expression in MCF-10A cells and found that overexpressing MCT-1 increased MnSOD but decreased p53 levels (Figure 4a), but knockdown of p53 (shp53) further increased the amount of MnSOD in MCT-1 oncogenic cells. Correspondingly, cellular ROS levels elevated by MCT-1 were enhanced via p53 knockdown (Figure 4b), but they were significantly suppressed by EGFR inactivation (AG1478). When H1299 cells (p53-null) were induced to re-express p53 (pCMV p53; Figure 4c), the MCT-1-induced MnSOD level was significantly repressed (lane 4). Moreover, the extent of the 
a

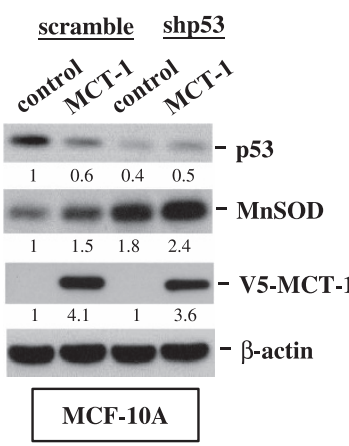

d

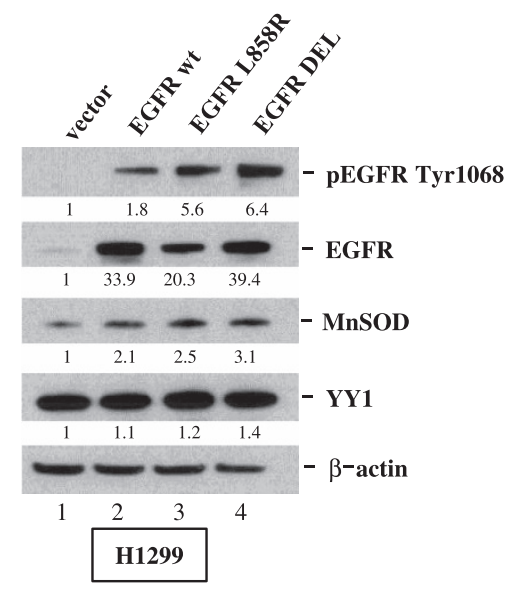

b

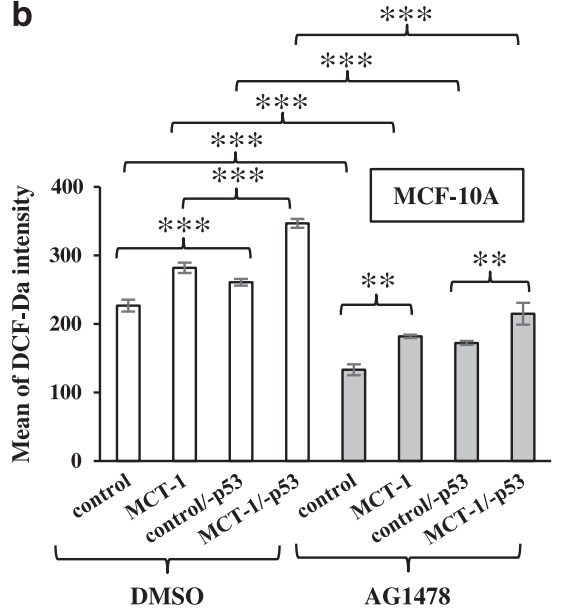

e

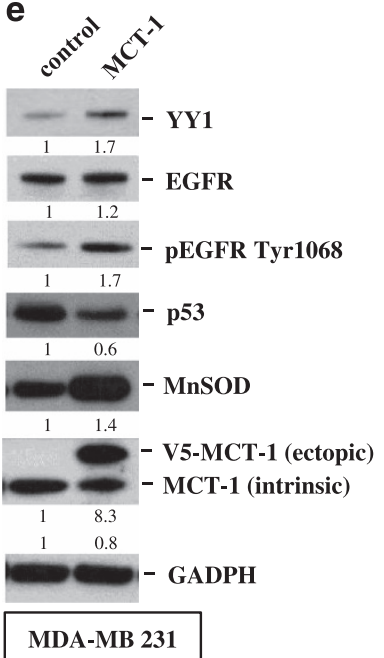

C

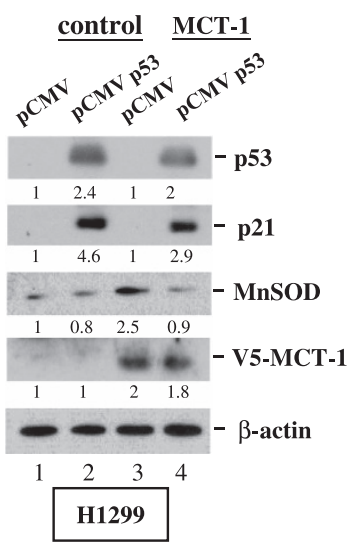

f

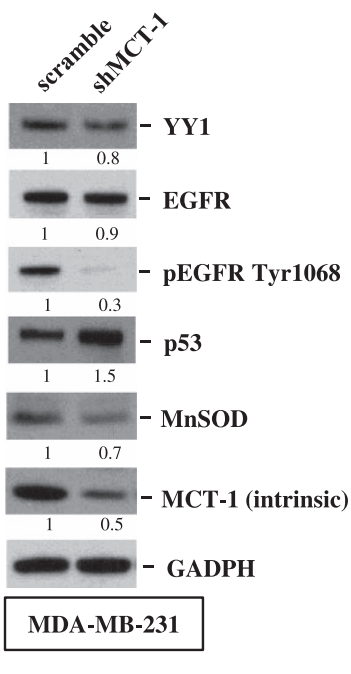

Figure 4. MnSOD is induced by MCT-1 overexpression, p53 knockdown and EGFR activation. (a) MnSOD expression was examined in MCF-10A cells (control and MCT-1) with (shp53) or without (scramble) p53 depletion. (b) Cellular ROS levels were analyzed in MCF-10A cells with different conditions of $\mathrm{p} 53$ and MCT-1 expression upon DMSO or AG1478 treatment. Data represent the mean \pm s.d. $(n=3)$. ${ }^{* *} P<0.01$; ${ }^{* * *} P<0.001$. (c) MnSOD levels were characterized in the H1299 cells (control and MCT-1) without (pCMV) or with p53 re-expression (pCMV p53). (d) The EGFR phosphorylation and MnSOD levels were assessed when $\mathrm{H} 1299$ cells were introducing empty vector, wild-type EGFR and EGFR-activating mutants (L858R and exon19 DEL). (e) MDA-MB231 cells with or without MCT-1 overexpression were assayed. (f) MDA-MB231 cells with or without MCT-1 knockdown were evaluated. The protein amounts were normalized to $\beta$-actin or GAPDH, and the phosphorylated EGFR levels were normalized to total EGFR levels before comparison with the comparative controls (lane 1).

increase in MnSOD corresponded to the advanced EGFR activity of the EGFR-activating mutants (L858R and exon19 deletion (DEL); lanes 3 and 4) compared with EGFRwt overexpression (lane 2) in H1299 cells (Figure 4d). Intriguingly, MnSOD was also induced in TNBC MDA-MB231 cells when MCT-1 overexpression induced YY1 induction, EGFR phosphorylation (Tyr1068) and decreased p53 levels (Figure 4e). Conversely, MnSOD was decreased, accompanied by EGFR de-phosphorylation (Tyr1068) and p53 accumulation when MCT-1 was targeted in MDA-MB231 cells (Figure 4f). Accordingly, MnSOD expression is determined by MCT-1 expression, p53 function and EGFR activation.

When MnSOD distribution was analyzed in the nuclear, mitochondrial (mito.) and cytosol fractions, we observed that the majority of MnSOD was located in the mitochondria, and a higher MnSOD level was found in MCT-1-overexpressing cells $(M)$ than in control A549 cells (C; Figure 5a, lane 4). Conversely, MnSOD levels were diminished equivalently to the extent of MCT-1 knockdown (clone \#3-9 and \#3-10; Figure 5b). MnSOD is a membrane-bound protein. ${ }^{45}$ Further evaluation of the MnSOD location in the cytoplasmic and membrane (mitochondria-containing) fractions revealed that only membrane-bound MnSOD was significantly elevated upon MCT-1 overexpression (Figure 5c), which may affect the redox metabolism of the oncogenic cells.

Although YY1 regulates mitochondrial complex I genes, ${ }^{46}$ and YY1 deficiency disrupts the structure and function of mitochondria, it is unknown whether YY1 affects MnSOD expression in cancer cells. In the subcellular fractionation analysis, YY knockdown (shYY1) in A549 cells showed a substantial inhibitory effect on EGFR and MnSOD in both the cytosolic and membrane compartments (Figure $5 d$, lanes 2 and 4) compared with scrambled knockdown. The MCT-1 promoter contains 10 putative YY1-binding sites (Supplementary Figure 2d). Consistent with this finding, loss of $Y Y 1$ reduced MCT-1, indicating that positive regulatory feedback exists between $\mathrm{YY} 1$ and MCT-1. Similar to the suppression of cellular MnSOD by silencing of YY1 (+shYY1; Supplementary Figure 2e), targeting EGFR (+shEGFR) also markedly inhibited the increase in MnSOD in MCT-1 oncogenic cells (Figure $5 \mathrm{e}$, lane 4). These data demonstrate, for the first time, that the MCT-1-YY1-EGFR pathway modulates MnSOD expression in cancer cells.

The phosphorylation of $\mathrm{p} 47^{\text {phox }}$ enhances NADPH oxidase (NOX) activation and superoxide production. ${ }^{47}$ Diphenyleneiodonium (DPI), 


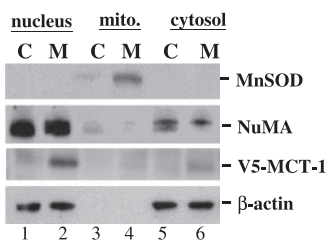

b

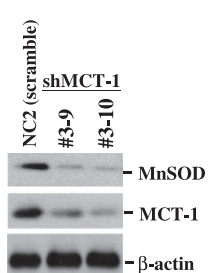

C

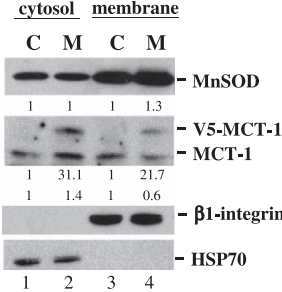

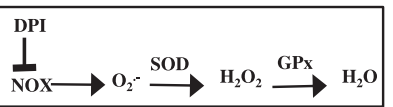

f

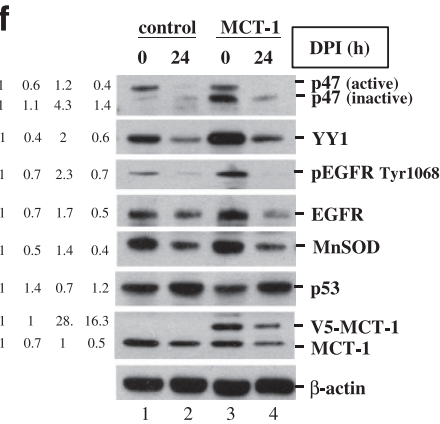

g

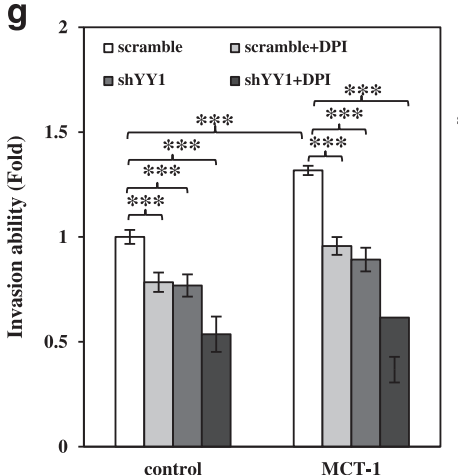

d

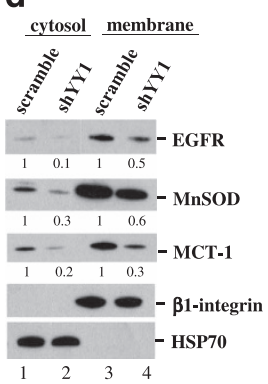

e

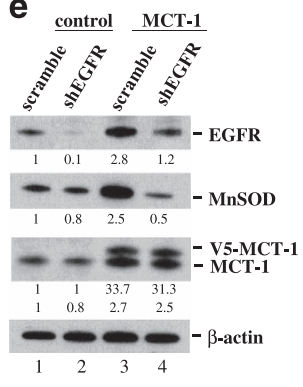

h

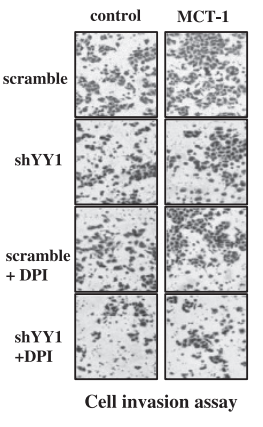

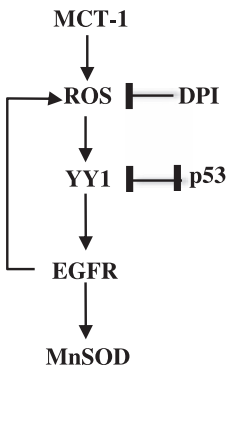

Figure 5. MCT-1 induces MnSOD expression and promotes cancer cell invasion via the YY1 pathway. The A549 cancer cells were studied. (a) Subcellular distributions of MnSOD, NuMA, MCT-1 and $\beta$-actin in the nucleus, mitochondria (mito.) and cytoplasm were characterized in control (C) and MCT-1-overexpressing (M) cells. (b) MnSOD expression levels were examined in different MCT-1-silencing clones (\#3-9 and \#3-10) and scrambled knockdown (NC2). (c) The distribution of MnSOD and MCT-1 in the cytosolic and membrane fractions was studied. HSP70 and $\beta 1$-integrin were the fraction markers. The amounts of cytosolic and membrane proteins were normalized to HSP70 and $\beta 1$-integrin, respectively, and then compared with scrambled knockdown (lanes 1 and 3). (d) The effects of YY1 knockdown (shYY1) on the distribution of EGFR, MnSOD, MCT-1 and HSP70 in the cytosol and membrane were studied. The cytosol and membrane protein amounts were normalized to HSP70 and $\beta 1$-integrin, respectively, and then compared with scrambled knockdown (lanes 1 and 3). (e) The effects of EGFR knockdown (shEGFR) on MnSOD and MCT-1 expression were assessed. The protein amounts were normalized to $\beta$-actin before comparison with scrambled control (lane 1). (f) The influence of DPI (an ROS inhibitor) on the expression of p47 ${ }^{\text {phox }}$ (active and inactive forms), YY1, EGFR, MnSOD and p53 was studied. The protein amounts were normalized to $\beta$-actin before comparison with the comparative control (lane 1). (g) Cell invasiveness affected by DPI treatment and YY1 knockdown was evaluated in different MCT-1 expression conditions. The data represent the mean \pm s.d. $(n=3)$. ${ }^{* * *} P<0.001$. (h) A proposed model of how MCT-1 overexpression promotes ROS generation and stimulates YY1-EGFR-MnSOD signaling, which can be suppressed by DPI and p53.

a ROS inhibitor, abolishes NOX-mediated superoxide $\left(\mathrm{O}_{2}^{-}\right)$ formation, which suppresses intracellular ROS levels. ${ }^{3}$ Intriguingly, we found that MCT-1 stimulated the expression of $\mathrm{p} 47^{\text {phox }}$, accompanied by enrichment of YY1, EGFR and MnSOD, in A549 cells (Figure $5 f$ ). However, DPI treatment not only reduced MCT-1 but also decreased p47 ${ }^{\text {phox }}$, YY1, EGFR and MnSOD (lane 4). These results suggest that $M C T-1$ enhances ROS generation and mitochondria superoxide formation, possibly by deregulating activities of oxidase (NOX) and antioxidant (MnSOD).

To explore the influence of $\mathrm{YY} 1$ and ROS on cancer cell invasiveness, we found that shYY1 and DPI suppressed the A549 cell invasiveness induced by MCT-1 (Figure $5 \mathrm{~g}$ ); thus, the combinatory effect (shYY1+DPI) strongly prohibited cancer cell invasion. The results from these studies reveal that MCT-1 overexpression induces ROS generation (Figure 5h), accompanied by YY1-EGFR-MnSOD signaling amplification and cancer invasiveness, which can be suppressed by DPI and p53 activity.

\section{Overexpression of MCT-1 promotes tumor progression and} transforms the tumor microenvironment

To determine whether the MCT-1 oncogenic pathway can alter the microenvironment(s) to benefit tumor progression, bioluminescent A549 cells were subcutaneously injected into $\mathrm{BALB} / \mathrm{c}$ nude mice, and tumors were allowed to develop for 8 weeks. Extensive tumor necrosis and increased tumor burdens were observed in mice-bearing MCT-1-overexpressing cells
(Figure 6a). An in vivo bioluminescence imaging system (IVIS) also detected a higher photon flux (Figure $6 \mathrm{~b}$ ), indicative of the advanced tumor growth underlying MCT-1 oncogenicity (Figure 6c).

A tumor necrotic cascade initiated by the oncogenic effect and oxidative stress would distort the function and structure of mitochondria. ${ }^{48,49}$ MCT-1 oncogenic activity promotes tumor necrosis, which may elicit an inflammatory response and a malignant microenvironment for tumor progression. ${ }^{50,51}$ To inspect changes in the tumor microenvironment, the angiogenesis marker CD31 (denoted by arrowheads), the cancer-associated fibroblast marker a-SMA (marked by asterisks) and the tumorassociated M2 macrophage marker CD163 (indicated by stars) were examined in an immunohistochemistry study (Figure $6 \mathrm{~d}$ ). Surprisingly, we observed that tumor angiogenesis (CD31), cancerassociated fibroblasts (a-SMA) and M2 macrophages (CD163) were all enriched, with increased YY1, p-EGFR (Tyr1068) and MnSOD expression but low p53 expression, in the MCT-1-overexpressing tumors compared with control tumors. These data reveal that MCT-1 promotes tumor progression, necrosis and the tumorpromoting activity of the microenvironment alongside the enhancement of YY, EGFR and MnSOD in vivo.

Clinical relevance of MCT-1, YY1, EGFR, MnSOD and p53 in human lung cancer

Lung cancer TissueScan qPCR arrays (OriGene Technologies, Inc.) were used to investigate the clinical relevance of MCT-1, YY1, 
a

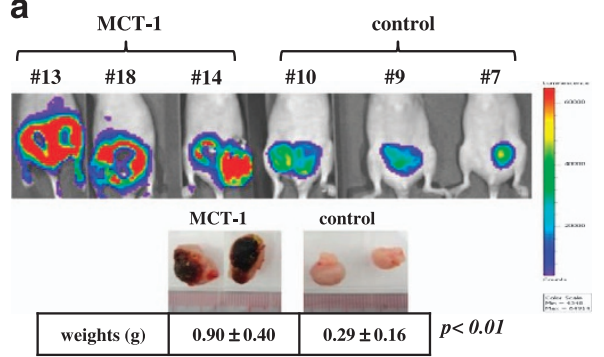

b

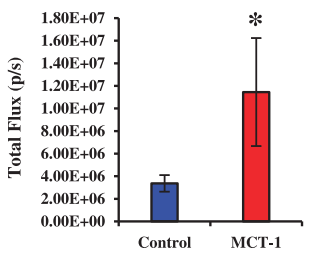

C

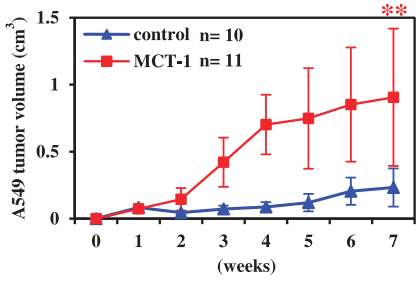

d MCT-1 4 YY-1 4 p-EGFR MnSOD $4 \quad \mathrm{p53} \downarrow$ CD31 个 $\alpha$-SMA $4 \quad$ CD163

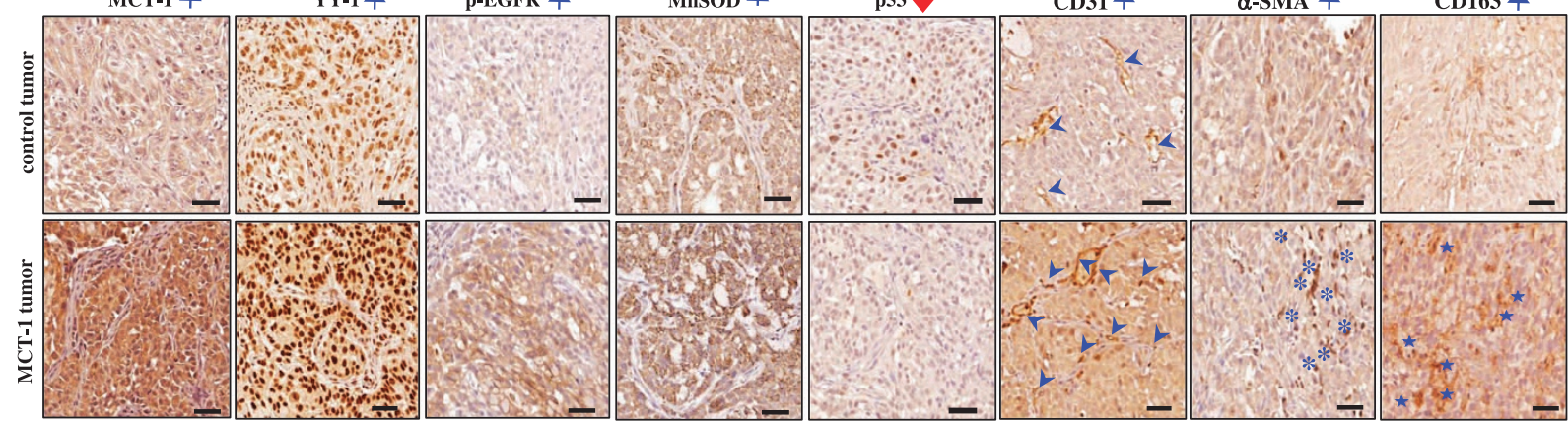

Figure 6. MCT-1 overexpression enhances A549 tumor progression. (a) Bioluminescent A549 cells were subcutaneously injected into nude mice, and tumors were allowed to develop for 8 weeks. Tumor expansion was detected by IVIS, and tumor weights were measured. (b) Photon flux detected by IVIS indicated tumorigenic outcomes at week 8. (c) Tumor growth was assessed weekly, and tumors in the xenograft mice injected with the control cells $(n=10)$ or MCT-1-overexpressing cells $(n=11)$ were compared. The data represent the mean \pm s.d. ${ }^{*} P<0.05$; ${ }^{* *} P<0.01$. (d) Immunohistochemistry results indicated the amounts of MCT-1, YY1, p-EGFR, MnSOD and p53 in the A549 tumors. Tumor angiogenesis (CD31, denoted by arrowheads) as well as the accumulation of myofibroblasts ( $\alpha$-SMA, indicated by asters) and tumor-promoting M2 macrophages (CD163, indicated by stars) were also characterized. Scale bar represents $50 \mu \mathrm{m}$.

EGFR, MnSOD and p53 expression in lung cancer patients. The results showed that MCT-1 mRNA levels were often induced in lung cancers $(n=124)$ at stage I $(77 \%, P<0.001)$, stage II $(73 \%$, $P<0.001)$ and stage III-IV (85\%, $P<0.001$; Figure 7a), which displayed a 1.5 -fold increase over the mean MCT- 1 mRNA level in normal lung tissue $(n=13)$. Overall, MCT- 1 was highly expressed in $79 \%$ of the patients with adenocarcinoma (82\%; Figure $7 b$ ), squamous cell carcinoma (79\%) and other cell type (small- and large-cell; 72\%) lung cancers. High MCT-1 levels were also identified in patients with lymph node metastasis $(N \geqslant 1, n=46$; $80 \%, P<0.001)$ and distal metastasis $(M=1, \quad n=12 ; 100 \%$, $P<0.001)$ compared with those without lymph node metastasis (78\%, $P<0.001)$ and distal metastasis $(77 \%, P<0.001)$.

Using the ONCOMINE (www.oncomine.org) lung cancer database and data-mining platform (Supplementary Figure 3), high MCT-1 mRNA levels were also detected in different types of lung cancers in Hou's data $(n=156)$ as well as in Okayama's lung adenocarcinoma data $(n=246, P=2.29 \mathrm{E}-19)$ and Wei's dataset $(n=50, P=1.10 \mathrm{E}-11)$. Further surveying the Okayama dataset in an 84 month follow-up study ( $n=83$; Figure $7 c),{ }^{52}$ high MCT- 1 levels were found to be significantly correlated with poor overall survival $(P<0.001)$ and low recurrence-free survival $(P<0.001)$ compared with the patients with low MCT-1 levels. In an analysis of the Okayama data (Figure 7d), high MCT-1 levels were linked with poor survival of the patients with EGFR mutations (exon19 (DEL) and L858R) more than low MCT-1 levels $(P<0.001)$. Accordingly, MCT-1 overexpression may be recognized as a biomarker for early detection and as a prognostic index in human lung carcinogenesis.

In contrast, only $50 \%$ of the cancer patients displayed high YY1 levels, and they were more prevalently identified in stage I $(67 \%, P<0.001)$ cancer than in stage $\|(37 \%, P<0.01)$ and stage III/IV $(41 \%, P<0.01)$ cancer relative to normal lung tissues (Supplementary Figure 4a). In addition, $70 \%$ of the patients expressed high EGFR levels ( $P<0.001$; Supplementary Figure $4 b$ ), and $71 \%$ of the patients exhibited MnSOD induction $(P<0.001$; Supplementary Figure $4 c$ ), whereas the majority of cancer patients (92\%) had p53 downregulation $(P<0.001)$ (Supplementary Figure $4 d$ ). The $\log _{2}$ ratio of MCT-1, p53, EGFR and MnSOD mRNA levels compared with $\beta$-actin mRNA levels further validated the significant differences between lung tumors $(T)$ and normal lung tissues (N; Supplementary Figure 4e).

To characterize the clinical relationship between MCT-1 and YY1, EGFR, MnSOD and p53, the average mRNA level of each gene in normal lung tissue was defined as the threshold. For a given gene, its expression level was dichotomized into 'low' and 'high'; it was 'high' if and only if it was higher than the normal tissue. Consequently, both MCT-1 and YY1 were found to often be induced in patients at stage I (Pearson correlation $+0.92, P<0.001$; Figure 7e), suggesting their strong association with the initial step of lung tumorigenesis. MCT-1 expression was largely positively associated with YY1 (Phi coefficient $+0.27, P=0.003$ ), EGFR (Phi coefficient $+0.43, P<0.001$ ) and MnSOD (Phi coefficient $+0.59, P<0.001$ ) in all stages of lung cancers (Figure 7f). However, MCT-1 had a strong negative correlation with p53 expression (Phi coefficient $-0.74, P<0.001$ ). As a result, enhanced MCT-1 activation is associated with increased YY1, EGFR and MnSOD but decreased p53 in lung carcinogenesis.

Furthermore, the association between MCT-1 with CD31, CD163 and a-SMA gene expression was characterized using Lung cancer TissueScan GPCR arrays (Figure $7 \mathrm{~g}$ ), and the results showed that MCT-1 was positively correlated with CD31 (Pearson coefficient $+0.31, P=0.03$ ), CD163 (Pearson coefficient $+0.37, P=0.01$ ) and a-SMA (Pearson coefficient $+0.32, P=0.03$ ) in stage III/IV lung cancers. However, MCT-1 elevation was less associated with CD31 (Pearson coefficient $+0.2, P=0.05$ ) and $a-S M A$ (Pearson coefficient $+0.25, P=0.02$ ) and was unrelated to CD163 (Pearson coefficient $+0.08, P=0.49$ ) in stage $\mathrm{I} / \mathrm{II}$ patients.

Based on an analysis of the ONCOMINE database (Figure 7h), high MCT-1 levels were positively correlated to CD31 (Pearson 
a

\begin{tabular}{|l|l|l|l|}
\hline \multicolumn{4}{|c|}{ MCT-1 mRNA level in lung cancer } \\
\hline Stage & MCT-1 high & MCT-1 low & p-value \\
\hline Normal & 0 & $13(100 \%)$ & \\
\hline I & $37(77 \%)$ & $11(23 \%)$ & $<0.001$ \\
\hline II & $22(73 \%)$ & $8(27 \%)$ & $<0.001$ \\
\hline III/IV & $\mathbf{3 9}(\mathbf{8 5} \%)$ & $7(15 \%)$ & $<0.001$ \\
\hline Total & $\mathbf{9 8}(\mathbf{7 9} \%)$ & $26(21 \%)$ & $<0.001$ \\
\hline
\end{tabular}

b

\begin{tabular}{|l|c|c|c|c|c|}
\hline \multicolumn{2}{|c|}{ Characteristics } & Total & MCT-1 high & MCT-1 low & p-value \\
\hline Tumor type & ADC & 56 & $46(82 \%)$ & $10(18 \%)$ & $<0.001$ \\
\hline \multirow{5}{*}{ N stage } & SCC & 43 & $34(79 \%)$ & $9(21 \%)$ & $<0.001$ \\
\hline \multirow{4}{*}{ others } & 25 & $18(72 \%)$ & $7(28 \%)$ & $<0.001$ \\
\hline M stage & 0 & 78 & $61(78 \%)$ & $17(22 \%)$ & $<0.001$ \\
\hline & $1-2$ & 46 & $37(80 \%)$ & $9(20 \%)$ & $<0.001$ \\
\hline Overall & 0 & 112 & $86(77 \%)$ & $26(23 \%)$ & $<0.001$ \\
\hline & 1 & 12 & $12(100 \%)$ & $0(0 \%)$ & $<0.001$ \\
\hline
\end{tabular}

* The mRNA level in the tumors with a $\geq 1.5$-fold increase over normal lung tissue recognized as high-expressio
* The mRNA level in the tumors with a 0.66 -fold reduction than normal lung tissue (set as 1 ) defined as low-

expression.

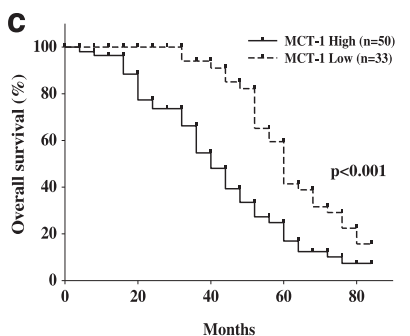

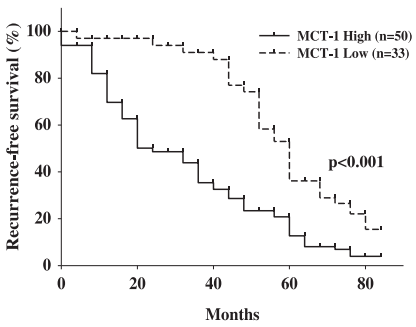

g e

\begin{tabular}{|l|c|c|}
\hline $\begin{array}{c}\text { MCT-1 high } \\
(\mathrm{n}=104)\end{array}$ & $\begin{array}{c}\text { Pearson } \\
\text { correlation }\end{array}$ & p-value \\
\hline YY1 (all stage) & +0.32 & 0.001 \\
\hline Stage I & +0.92 & $<0.001$ \\
\hline Stage II & +0.21 & 0.28 \\
\hline Stage III/IV & +0.008 & 0.96 \\
\hline
\end{tabular}

f

\begin{tabular}{|l|c|c|}
\hline $\begin{array}{l}\text { MCT-1 } \\
(\mathbf{n}=\mathbf{1 2 4})\end{array}$ & $\begin{array}{c}\text { r-value } \\
(\phi)\end{array}$ & p-value \\
\hline YY1 & +0.27 & 0.003 \\
\hline EGFR & $+\mathbf{+ 0 . 4 3}$ & $<0.001$ \\
\hline MnSOD & $+\mathbf{+ 0 . 5 9}$ & $<0.001$ \\
\hline p53 & $-\mathbf{0 . 7 4}$ & $<0.001$ \\
\hline
\end{tabular}

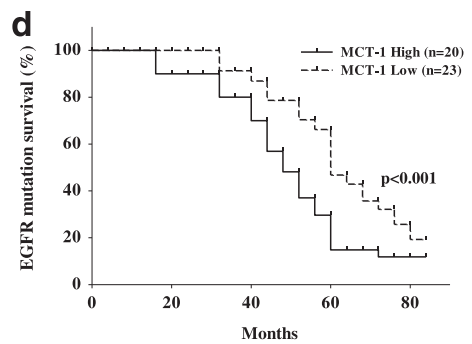

h

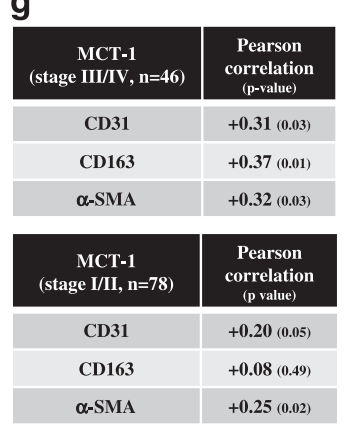

Figure 7. Clinical relevance of MCT-1 and its relationship with YY1, EGFR, MnSOD, CD31, CD163 and $\alpha$-SMA in human lung cancer. (a) MCT-1 mRNA levels in lung cancer tissue $(n=124)$ and normal lung tissue $(n=13)$ were studied with the TissueScan arrays. The MCT-1 mRNA level in each tumor sample was normalized to the $\beta$-actin mRNA level and then calibrated to the average MCT-1 mRNA level in normal lung tissue. $X^{2}$ tests were used to evaluate the significance of MCT-1 overexpression at different tumor stages compared with the normal tissue. (b) MCT-1 mRNA levels were assessed in different tumor types, in lymph node metastasis $(N \geqslant 1)$ and in distant metastasis $(M=1)$ using $X^{2}$ tests. (c) The correlation between MCT-1 expression levels and overall survival and recurrence-free survival $(n=83)$ were analyzed using the Okayama dataset in the ONCOMINE database. (d) The overall survival of patients with an EGFR mutation associated with MCT-1 expression levels was analyzed in the Okayama dataset $(n=43)$. (e) Pearson coefficient correlation defines the linkage between MCT-1 overexpression and YY1 $(n=104)$. (f) The Phi coefficient shows the relationship of MCT-1 with YY1, EGFR, MnSOD and p53 in lung cancer patients ( $n=124)$. (g) Pearson coefficient correlation reveals the association between MCT- 1 with CD31, CD163 and $\alpha$-SMA in the lung cancers at stage III/IV ( $n=46)$ and at stage I/II $(n=78)$. (h) Pearson coefficient correlation indicates the association between MCT-1 and CD31, CD163 and $\alpha$-SMA in different types and stages of lung cancer in the ONCOMINE database (Bild, $n=26$; Weiss, $n=217$; Okayama, $n=207$ ).

correlation $+0.62, P=0.01$ ), a-SMA (Pearson correlation +0.15 , $P=0.03$ ) and CD163 (Pearson correlation $+0.35, P<0.001$ ) expression in stage III/IV and different types of lung cancers. Collectively, MCT-1 overexpression is implicated in lung carcinogenesis and development of the tumor microenvironment.

\section{DISCUSSION}

Targeted therapies have achieved notable successes in some cancers. However, many targeted therapies are highly toxic to normal cells, and most patients experience relapse due to genetic mutation and tumor heterogeneity, whereby the tumors contain therapy-resistant stem cells that have adopted alternative and compensatory pathways. A combination of a ROS inhibitor with the suppression of an oncogenic pathway may be a low-toxic 'broad-spectrum' therapeutic approach that simultaneously targets many key pathways and mechanisms to alter the tumor microenvironment and prevent metastasis. ${ }^{53}$

We now demonstrate that oncogenic MCT-1 activation elevates ROS generation and amplifies YY1-EGFR-MnSOD signaling, accompanied by tumor promotion and a malignant microenvironment. Clinical data confirm the important connection between MCT-1 activity and YY1, EGFR and MnSOD, as well as the development of malignant microenvironments in lung carcinoma. Targeting the MCT-1 pathway may alter oxidative metabolism and reduce tumor aggressiveness.

Elevated ROS-mediated regulation of the T-cell immune response in the microenvironment is related to tumor-induced immunosuppression. ${ }^{54}$ Thus, T-cell-based cancer therapy combined with a ROS scavenger has been considered a promising strategy to improve T-cell immunity. ROS released from cancer cells potentially promote stromal fibroblast transformation into myofibroblasts that support tumor progression and dissemination. ${ }^{55}$ Thus, using antioxidants as an adjuvant therapy to modify tumor immunity and the microenvironment may lead to beneficial clinical outcomes.

MCT-1 enhances EGFR expression, possibly via a cooperative increase in YY1 (Figure 1) and SP1 as previously described. ${ }^{28,56,57}$ MicroRNA-34a (miR-34a) is induced by p53 and directly targets YY1 to inhibit YY1-induced and EGFR-mediated carcinogenesis 
and metastasis. ${ }^{58-61}$ Loss of p53 amplifies MCT-1-YY1-EGFR signaling (Supplementary Figure 1) and induces MnSOD (Figure 4a), which may produce excessive ROS that enhance genetic mutation and tumor progression. Furthermore, MCT-1 may potentiate tumor angiogenesis by antagonizing the p53-miR-34a axis and inhibiting the angiogenesis inhibitor thrombospondin-1 (TSP1). ${ }^{24,62}$

Under oncogenic stress, mitochondrial ROS overproduction can accelerate mutagenesis, which amplifies tumorigenic signaling and increases metastatic potential. ${ }^{63}$ Overexpression or activatingmutation of EGFR promotes tumor progression and metastasis. ${ }^{64}$ EGFR activity is implicated in DNA repair. ${ }^{65}$ Therefore, advanced ROS generation and increased cell survival via the combined effect of MCT-1 and EGFR may mis-repair oxidative DNA damage and promote growth of gene-mutated cells.

EGFR overexpression and mutation are frequently identified in metastatic non-small-cell lung cancer and are associated with poor prognosis. ${ }^{66}$ L858R is the most prevalent EGFR tyrosine kinase activating mutation. Mutation of the kinase domain in EGFR leads to a ligand-independent tyrosine kinase activation. EGFR activation is crucial for ROS production because deactivation of EGFR by AG1478 suppresses the generation of ROS (Figure 4b), explaining why ROS levels are higher in L858R mutant than wildtype EGFR cells (Figure 3c). The cells expressing wild-type EGFR have a higher ability to repair cisplatin- and IR-induced DNA damage than the L858R-mutant-expressing cells; ${ }^{67}$ therefore, the intracellular ROS levels are reduced because wild-type EGFR repairs the oxidative DNA damage.

Compared with other subtypes of breast cancer, EGFR expression, gene amplification and mutations are more frequently identified and associated with poor prognosis in TNBCs. ${ }^{42,68,69}$ For example, activating mutations in the EGFR gene (exon19 deletion and L858R and T790M mutations) are present in the tumors of TNBC patients. ${ }^{68,70}$ Therefore, EGFR may also be a therapeutic target in TNBCs.

Enhanced MCT-1 activity induces the superoxide scavenger MnSOD and ROS generators, including NADPH (p47 ${ }^{\text {phox }}$; Figure 5) and Shc (p66), ${ }^{29}$ explaining why the MCT-1 oncogenic cells survive and are capable of adapting to the oxidative environment (Figures $2 \mathrm{e}$ and $3 \mathrm{~b}$ ). Here, we show that MnSOD expression is stimulated by MCT-1 overexpression, p53 deficiency and EGFR activation (Figures 4 and 5), which may be due to the overproduction of mitochondrial $\mathrm{O}_{2}^{-}$in these cellular backgrounds. Typically, cytoplasmic p53 translocation into mitochondria interacts with MnSOD to control the mitochondrial ROS level. ${ }^{71}$ Therefore, the $\mathrm{p} 53$ function suppressed by MCT-1 could lead to MnSOD induction and ROS promotion in carcinogenic cells (Figures $4 \mathrm{a}$ and b). Upregulation of MnSOD sustains the Warburg effect via mitochondrial ROS generation and AMP-activated kinase activation, which increases the metabolic shift to glycolysis and maintains tumor aggressiveness. ${ }^{15}$ Targeted suppression of MCT-1, YY1 and EGFR capably effectively suppress MnSOD expression in cancer cells (Figures $4 f, 5 b, d$ and e), emphasizing that the amplification of MnSOD signaling via the MCT-1-YY1-EGFR network may determine cancer cell proliferation, invasion and metastasis. Thus, scavenging mitochondrial superoxide formation by modulating the MCT-1-YY1-EGFR-MnSOD axis together with a ROS inhibitor that reduces intracellular and extracellular oxidative stresses might alleviate carcinoma metabolism and metabolic reprogramming of the microenvironment in aggressive tumor.

\section{MATERIALS AND METHODS}

Cell lines

MCF-10A cells were cultured in DMEM/F-12 complete medium. ${ }^{27}$ A549, H1299 and MDA-MB231 cells were cultured in RPMI 1640 medium. ${ }^{29}$ V5-tagged MCT-1/pLXSN, pCMV p53 and the empty vehicles (pLXSN and pCMV) were transfected into cells as previously described. ${ }^{29}$
The YY1 gene was cloned from A549 cells using a PCR cloning strategy (forward primer: 5'-ATGGCCTCGGGCGACACCCTCTACATCGCCAC-3' and reverse primer: 5'-CTGGTTGTITIGGCCTTAGCATGTGTTAAGA-3) and inserted into a pLHCX vector with a $3 \times$ FLAG peptide (DYKDDDDK) at the carboxyl terminus. Transfectants carrying pLHCX empty vehicle or FLAG-tagged YY1 vector were maintained in medium containing $50 \mu \mathrm{g} / \mathrm{ml}$ hygromycin.

The pBABE plasmid carrying wild-type EGFR or a mutant EGFR (L858R) gene (Addgene, Cambridge, MA, USA) were transfected into MCF-10A cells, and cells were cultured in $0.5 \mu \mathrm{g} / \mathrm{ml}$ puromycin-containing medium. The H1299 cell variants with wild-type EGFR and EGFR-activating mutants (L858R and exon19 deletion (DEL)) were established as previously described. $^{72}$

MCT-1 expression was suppressed by SureSilencing pGeneClip MCT-1 shRNA (SABiosciences, Valencia, CA, USA) as previously described. ${ }^{30}$ The cells were further transfected with scrambled shRNA or pMKO.1 puro p53 shRNA 2 (Addgene) to deplete p53. Similarly, YY1 and EGFR were, respectively, inhibited in cells using SureSilencing YY1 shRNA (SABiosciences) and EGFR shRNA (SABiosciences) with a TransIT-LT1 transfection reagent (Mirus Bio LLC, Madison, WI, USA) according to the manufacturer's instructions.

Antibodies (Abs)

Abs recognizing p53 (DO-1; Santa Cruz Biotechnology, Santa Cruz, CA), GAPDH, $\beta$-actin (Abcam, Cambridge, MA, USA), CD31 (BD Pharmingen, San Diego, CA, USA), MnSOD (Enzo Life Sciences, Inc., Farmingdale, NY, USA), p $47^{\text {phox }}$ (Signalway Antibody, Baltimore, MD, USA) and integrin beta-1 (CD29; BD Transduction Laboratories, Lexington, KY, USA) were purchased. Abs against phospho-p53 (Ser15), phospho-EGFR (Tyr1068), EGFR and H2AX (Ser139) were obtained from Cell Signaling Technology (Danvers, MA, USA). The Abs against V5-epitope (Invitrogen, Waltham, MA, USA) and intrinsic MCT-1 (N1C3) GeneTex (Irvine, CA, USA) were purchased. Western blot analysis was performed as previously described. ${ }^{30}$

\section{Apoptosis analysis}

A FITC-Annexin V Apoptosis Detection Kit (BD Biosciences, San Jose, CA, USA) was used to analyze apoptosis. The cells were incubated in complete medium with $100 \mu \mathrm{m}$ hydrogen peroxide $\left(\mathrm{H}_{2} \mathrm{O}_{2}\right)$ for $24 \mathrm{~h}$ or pre-treated with $5 \mu \mathrm{M}$ AG1478 for $1 \mathrm{~h}$ before $\mathrm{H}_{2} \mathrm{O}_{2}$ exposure as indicated. ${ }^{73}$ All the cells were re-suspended in $1 \times$ binding buffer $\left(1 \times 10^{6}\right.$ cells $\left./ \mathrm{ml}\right)$ and reacted with Annexin V-FITC for $15 \mathrm{~min}$. Propidium iodide was used as a counterstain to discriminate necrotic/dead cells. The results were analyzed using a BD FACSCalibur flow cytometer (Becton-Dickinson, San Jose, CA, USA). Annexin V-FITC binding was detected by flow cytometry ( $E x=488 \mathrm{~nm}$; $\mathrm{Em}=350 \mathrm{~nm}$ ) using a FITC signal detector (FL1), and propidium iodide staining was detected using a phycoerythrin emission signal detector (FL2). The results were analyzed using BD CellQuest Pro Analysis software (BD Biosciences).

An In Situ Cell Death Detection kit (Roche, Mannheim, Germany) was used to evaluate DNA fragmentation in apoptotic cells with terminal deoxynucleotidyl transferase dUTP nick-end labeling (TUNEL) followed by flow cytometry, according to the manufacturer's instructions.

Promoter activity assay

A YY1 promoter $(-1514$ to +54$)$ fragment was cloned from A549 cells and inserted into a pGL3-Luciferase basic vector between Nhel and Xhol restriction sites. The EGFR promoter vector was constructed as previously described. ${ }^{74}$ The $\mathrm{p} 53$ promoter fragment $(-188$ to +23$)$ was cloned from MCF-10A cells by PCR amplification using the forward primer $5^{\prime}$-cga gctcgtcggcgagaatcctgact- $3^{\prime}(-188$ to -170$)$ and the reverse primer $5^{\prime}$-gga agcttGGACGGTGGCTCTAGACTTा-3' (+3 to +23 ) and then cloned into a pGL3-Luciferase vector between Sacl and Hind/ll restriction sites. Luciferase reporter activity was measure as previously described. ${ }^{27}$

Examination of intracellular ROS and mitochondrial superoxide DCFH-DA (2',7'-dichlorofluorescein diacetate; Sigma-Aldrich, St. Louis, MO) and MitoSOX Red (Molecular Probes, Eugene, OR, USA) were used to measure cellular ROS and mitochondrial superoxide, respectively. Cells were incubated with $50 \mu \mathrm{m} \mathrm{DCFH-DA}$ or $5 \mu \mathrm{m}$ MitoSOX at $37^{\circ} \mathrm{C}$ for $30 \mathrm{~min}$. The emitted DCF and MitoSOX fluorescence were quantified using a FACSCalibur flow cytometer (Becton-Dickinson) with excitation/emission wavelengths of $485 / 530 \mathrm{~nm}$ to measure DCF in the FL1 channel and with 
excitation/emission wavelengths of $510 / 580 \mathrm{~nm}$ to measure oxidized MitoSOX Red in the FL2 channel.

\section{Tumor progression and immunohistochemistry study}

The A549 cells carrying the pcDNA3.1/luciferase vector $\left(1 \times 10^{6} / 100 \mu \mathrm{l}\right.$ PBS) were inoculated subcutaneously (s.c.) into 6-week-old female BALB/c nude mice (BALB/CAnN.Cg-Foxn $1^{\text {nu }} /$ CrINarl; $n=11$ ), and tumors were allowed to develop for 8 weeks. A randomized method was used to assign the mice into the experimental groups (control and MCT-1). The animal studies were conducted in accordance with the Animal Use Protocol approved by the National Health Research Institutes (NHRI-IACUC-104020-A). Tumor tissues were processed for immunohistochemistry analysis as previously described. ${ }^{27}$ Luciferin (150 mg/kg; PerkinElmer, Waltham, MA, USA) was intraperitoneally injected into mice to detect tumor progression using a Xenogen IVIS 200 bioluminescence imaging system (Caliper LifeSciences, Hopkinton, MA, USA).

The Abs for immunohistochemistry were diluted as follows: MCT-1, 1:500 (GeneTex, GTX117793); YY1, 1:400 (GeneTex, GTX62783); CD31, 1:200 (Abcam, ab28364); CD163, 1:100 (Abcam, ab189915); aSMA, 1:4000 (GeneTex, GTX112862); MnSOD, 1:2000 (Enzo Life Sciences, Inc., ADI-SOD-111-D); p53, 1:100 (Millipore, DAM1698716, Billerica, MA, USA); and phospho-EGFR, 1:100 (Cell Signaling Technology, \#4407S).

\section{Quantitative real-time PCR}

Real-time PCR was conducted using SYBR Green Master Mix and analyzed using a LightCycler PCR detection system (ABI PRISM-7900) as described previously. ${ }^{29}$ The primers for MCT-1 (forward: 5'-AGGCATTATCTTCAT GCTGTCA-3'; reverse: 5'-AATGATGGGCTGTGGCATAT-3'), YY1 (forward: 5'-GGAACAAGGGCTCTCA AACC-3'; reverse: $5^{\prime}$-CCCGGCAAGTGTGAGTG-3'), EGFR (forward: 5'-CTCCGTTTCTTCTTTGC CCAG-3'), p53 (forward: 5'-TTCCTC TTCCTACAGTACTCC-3'; reverse: $5^{\prime}$-GACGCGGGTGC CGGGCGG-3'), MnSOD (forward: 5'-AGCTATTTGGAATGTAATCAACTGG-3'; reverse: 5'-TAAGCAACAT CAAGAAATGCTACA-3'), CD31 (forward: 5'-TAATACAACATCCACGAGG GTC-3'; reverse: 5'-CTGACAGTGTCTTGAGTGGG-3'), CD163 (forward: 5'-CCG GGAGATGAATTCTTGCCT-3'; reverse: 5'-AGACACAGAAATTAGTTCAGCA GCA-3'), a-SMA (forward: 5'-GCTAGAGACAGAGAGGAGCAGG-3'; reverse: $5^{\prime}$-CTCTCTGTCCACCTTCCAGC- $3^{\prime}$ ) and $\beta$-actin (forward: $5^{\prime}$-CACCAGGGCGTG ATG GTGGG-3'; reverse: $5^{\prime}$-GATGCCTCTCTTGCTCTGGGC-3') were designed according to the NCBI Probe database.

The half-life of YY1 mRNA was measured when the A549 cells were treated with $5 \mu \mathrm{g} / \mathrm{ml}$ actinomycin $D$ (Sigma-Aldrich) for various times, and the remaining YY1 mRNA was analyzed by quantitative real-time PCR.

\section{Subcellular fractionation}

A Mitochondria/Cytosol Fractionation Kit (Abcam, Cambridge, MA, USA) was used to perform subcellular fraction. Cells $\left(5 \times 10^{6}\right.$ cells $)$ were incubated in $1 \times$ Cytosol Extraction Buffer Mix containing DTT and protease inhibitors, homogenized in an ice-cold Dounce tissue grinder and centrifuged at $700 \mathrm{~g}$ in a microcentrifuge for $10 \mathrm{~min}$ at $4^{\circ} \mathrm{C}$. The nuclear pellet was kept, and the supernatant was further centrifuged at $10000 \mathrm{~g}$ for $30 \mathrm{~min}$ at $4{ }^{\circ} \mathrm{C}$. The supernatant (cytosolic fraction) was collected, and the pellet (mitochondria) was re-suspend with Mitochondrial Extraction Buffer to isolate mitochondrial proteins. Nuclear proteins were extracted with $1 \times$ SDS-PAGE sample buffer.

A Subcellular Protein Fractionation kit (Thermo Fisher Scientific Inc., Waltham, MA, USA) was used to characterize cytosolic and membrane proteins. Cells $\left(5 \times 10^{6}\right.$ cells) were incubated in cytoplasmic extraction buffer on ice for $10 \mathrm{~min}$. The supernatant was centrifuged at $500 \mathrm{~g}$ for $5 \mathrm{~min}$ to collect the cytosolic fraction. The pellet was incubated in membrane extraction buffer on ice for $10 \mathrm{~min}$ and centrifuged at $3000 \mathrm{~g}$ for $5 \mathrm{~min}$ to collect the membrane fraction.

\section{Cell invasion assay}

Cell invasiveness was examined using Corning BioCoat Tumor Cell Invasion Systems (Corning, Corning, NY, USA). The cells $\left(5 \times 10^{4}\right)$ were incubated with serum-free medium and $6 \mu \mathrm{m}$ DPI (Sigma-Aldrich) or DMSO in the top insert and with $10 \%$ FBS-containing medium in the bottom chamber for $24 \mathrm{~h}$. Cells invading to the lower chamber were fixed with methanol, stained with crystal violet and counted using a microscope and the $\times 20$ objective lens.
Gene expression levels in TissueScan Lung Cancer tissue array TissueScan Lung Cancer Tissue qPCR Panels (II, III and V; OriGene Technologies, Inc., Rockville, MD, USA) were used to analyze MCT-1, YY1, EGFR, MnSOD, p53, CD31, CD163 and a-SMA mRNA levels by quantitative RT-PCR analysis. The relative mRNA levels were calculated using the formula: $\Delta C_{T}=C_{t}$ normal tissue group $-C_{t}$ cancer group. The fold change in each gene was calculated using the formula $2^{-\Delta C T}$. Clinical studies were approved by the Institutional Review Board (IRB) of the National Health Research Institutes (EC1031216-W).

\section{Statistics}

Student's $t$-test was used to compare the mean of the control group and the experimental groups. $X^{2}$ tests were used to assess differences between cancer stages and different cancer types and normal tissues. The Phi coefficient was used to evaluate the relationship between high expression of MCT-1 and YY1. Pearson's coefficient correlation was used to evaluate the relationship between MCT-1 and YY1, EGFR, MnSOD, p53, CD31, CD163 and a-SMA. Correlations between MCT-1 expression levels and overall survival, recurrence-free survival and EGFR mutations in the Okayama data set were analyzed using Student's $t$-test. A $P$-value $<0.05$ was considered statistically significant.

\section{CONFLICT OF INTEREST}

The authors declare no conflict of interest.

\section{ACKNOWLEDGEMENTS}

We thank Dr Yi-Rong Chen for providing the H1299 cell lines expressing wild-type EGFR and L858R and exon19 deletion mutant EGFR. This work was funded by Ministry of Science and Technology of Taiwan (MOST) 103-2320-B-400-012 and 105-2320-B-400-003 and supported by the National Health Research Institutes of Taiwan MG-105-PP-05 and NHRI-EX105-10523 El.

\section{REFERENCES}

1 Hurd TR, DeGennaro M, Lehmann R. Redox regulation of cell migration and adhesion. Trends Cell Biol 2012; 22: 107-115.

2 Gupta SC, Hevia D, Patchva S, Park B, Koh W, Aggarwal BB. Upsides and downsides of reactive oxygen species for cancer: the roles of reactive oxygen species in tumorigenesis, prevention, and therapy. Antioxid Redox Signal 2012; 16: 1295-1322.

3 Wind S, Beuerlein K, Eucker T, Muller $\mathrm{H}$, Scheurer $\mathrm{P}$, Armitage ME et al. Comparative pharmacology of chemically distinct NADPH oxidase inhibitors. $\mathrm{Br} J$ Pharmacol 2010; 161: 885-898.

4 Costa A, Scholer-Dahirel A, Mechta-Grigoriou F. The role of reactive oxygen species and metabolism on cancer cells and their microenvironment. Semin Cancer Biol 2014; 25: 23-32.

5 Sosa V, Moline T, Somoza R, Paciucci R, Kondoh H, ME, LL. Oxidative stress and cancer: an overview. Ageing Res Rev 2013; 12: 376-390.

6 Toullec A, Gerald D, Despouy G, Bourachot B, Cardon M, Lefort S et al. Oxidative stress promotes myofibroblast differentiation and tumour spreading. $E M B O \mathrm{Mol}$ Med 2010; 2: 211-230.

7 Xia C, Meng Q, Liu LZ, Rojanasakul Y, Wang XR, Jiang BH. Reactive oxygen species regulate angiogenesis and tumor growth through vascular endothelial growth factor. Cancer Res 2007; 67: 10823-10830.

8 Chen ZJ. Ubiquitin signalling in the NF-kappaB pathway. Nat Cell Biol 2005; 7: 758-765.

9 Shi X, Zhang Y, Zheng J, Pan J. Reactive oxygen species in cancer stem cells. Antioxid Redox Signal 2012; 16: 1215-1228.

10 Liu Y, Elf SE, Miyata Y, Sashida G, Liu Y, Huang G et al. p53 regulates hematopoietic stem cell quiescence. Cell Stem Cell 2009; 4: 37-48.

11 Drane P, Bravard A, Bouvard V, May E. Reciprocal down-regulation of p53 and SOD2 gene expression-implication in p53 mediated apoptosis. Oncogene 2001; 20: 430-439.

12 Dhar SK, Xu Y, Chen Y St, Clair DK. Specificity protein 1-dependent p53-mediated suppression of human manganese superoxide dismutase gene expression. J Biol Chem 2006; 281: 21698-21709.

13 Hussain SP, Amstad P, He P, Robles A, Lupold S, Kaneko I et al. p53-induced up-regulation of MnSOD and GPx but not catalase increases oxidative stress and apoptosis. Cancer Res 2004; 64: 2350-2356. 
14 Dhar SK, Tangpong J, Chaiswing L, Oberley TD St, Clair DK. Manganese superoxide dismutase is a p53-regulated gene that switches cancers between early and advanced stages. Cancer Res 2011; 71: 6684-6695.

15 Hart PC, Mao M, de Abreu AL, Ansenberger-Fricano K, Ekoue DN, Ganini D et al. MnSOD upregulation sustains the Warburg effect via mitochondrial ROS and AMPK-dependent signalling in cancer. Nat Commun 2015; 6: 6053.

16 Mantymaa P, Siitonen T, Guttorm T, Saily M, Kinnula V, Savolainen ER et al. Induction of mitochondrial manganese superoxide dismutase confers resistance to apoptosis in acute myeloblastic leukaemia cells exposed to etoposide. $\mathrm{Br} J$ Haematol 2000; 108: 574-581.

17 Loo SY, Hirpara JL, Pandey V, Tan TZ, Yap CT, Lobie PE et al. Manganese superoxide dismutase expression regulates the switch between an epithelial and a mesenchymal-like phenotype in breast carcinoma. Antioxid Redox Signal 2016; 25: 283-299.

18 Li S, Mao Y, Zhou T, Luo C, Xie J, Qi W et al. Manganese superoxide dismutase mediates anoikis resistance and tumor metastasis in nasopharyngeal carcinoma. Oncotarget 2016; 7: 32408-32420.

19 Bae IH, Park MJ, Yoon SH, Kang SW, Lee SS, Choi KM et al. Bcl-w promotes gastric cancer cell invasion by inducing matrix metalloproteinase-2 expression via phosphoinositide 3-kinase, Akt, and Sp1. Cancer Res 2006; 66: 4991-4995.

20 Kim EM, Kim J, Park JK, Hwang SG, Kim WJ, Lee WJ et al. Bcl-w promotes cell invasion by blocking the invasion-suppressing action of Bax. Cell Signal 2012; 24: 1163-1172.

21 Reinert LS, Shi B, Nandi S, Mazan-Mamczarz K, Vitolo M, Bachman KE et al. MCT-1 protein interacts with the cap complex and modulates messenger RNA translational profiles. Cancer Res 2006; 66: 8994-9001.

22 Schleich S, Strassburger K, Janiesch PC, Koledachkina T, Miller KK, Haneke K et al. DENR-MCT-1 promotes translation re-initiation downstream of uORFs to control tissue growth. Nature 2014; 512: 208-212.

23 Skabkin MA, Skabkina OV, Dhote V, Komar AA, Hellen CU, Pestova TV. Activities of Ligatin and MCT-1/DENR in eukaryotic translation initiation and ribosomal recycling. Genes Dev 2010; 24: 1787-1801.

24 Mazan-Mamczarz K, Hagner PR, Corl S, Srikantan S, Wood WH, Becker KG et al. Post-transcriptional gene regulation by HuR promotes a more tumorigenic phenotype. Oncogene 2008; 27: 6151-6163.

25 Kasiappan R, Shih HJ, Chu KL, Chen WT, Liu HP, Huang SF et al. Loss of p53 and MCT-1 overexpression synergistically promote chromosome instability and tumorigenicity. Mol Cancer Res 2009; 7: 536-548.

26 Hsu HL, Choy CO, Kasiappan R, Shih HJ, Sawyer JR, Shu CL et al. MCT-1 oncogene downregulates p53 and destabilizes genome structure in the response to DNA double-strand damage. DNA Repair (Amst) 2007; 6: 1319-1332.

27 Kasiappan R, Shih HJ, Wu MH, Choy C, Lin TD, Chen L et al. The antagonism between MCT-1 and p53 affects the tumorigenic outcomes. Mol Cancer 2010; 9: 311.

$28 \mathrm{Hsu}$ HL, Shi B, Gartenhaus RB. The MCT-1 oncogene product impairs cell cycle checkpoint control and transforms human mammary epithelial cells. Oncogene 2005; 24: 4956-4964.

29 Shih HJ, Chen HH, Chen YA, Wu MH, Liou GG, Chang WW et al. Targeting MCT-1 oncogene inhibits Shc pathway and xenograft tumorigenicity. Oncotarget 2012; 3 : 1401-1415.

30 Shih HJ, Chu KL, Wu MH, Wu PH, Chang WW, Chu JS et al. The involvement of MCT-1 oncoprotein in inducing mitotic catastrophe and nuclear abnormalities. Cell Cycle 2012; 11: 934-952.

$31 \mathrm{Wu} \mathrm{MH}$, Chen YA, Chen HH, Chang KW, Chang IS, Wang LH et al. MCT-1 expression and PTEN deficiency synergistically promote neoplastic multinucleation through the Src/p190B signaling activation. Oncogene 2014; 33: 5109-5120.

32 Chakraborty S, Li L, Puliyappadamba VT, Guo G, Hatanpaa KJ, Mickey B et al. Constitutive and ligand-induced EGFR signalling triggers distinct and mutually exclusive downstream signalling networks. Nat Commun 2014; 5: 5811.

33 Hashimoto A, Kurosaki M, Gotoh N, Shibuya M, Kurosaki T. Shc regulates epidermal growth factor-induced activation of the JNK signaling pathway. J Biol Chem 1999; 274: 20139-20143.

34 Rozakis-Adcock M, McGlade J, Mbamalu G, Pelicci G, Daly R, Li W et al. Association of the Shc and Grb2/Sem5 SH2-containing proteins is implicated in activation of the Ras pathway by tyrosine kinases. Nature 1992; 360: 689-692.

35 Agarwal N, Dancik GM, Goodspeed A, Costello JC, Owens C, Duex JE et al. GON4L drives cancer growth through a YY1-androgen receptor-CD24 axis. Cancer Res 2016; 76: 5175-5185.

36 Kaufhold S, Garban H, Bonavida B. Yin Yang 1 is associated with cancer stem cell transcription factors (SOX2, OCT4, BMI1) and clinical implication. J Exp Clin Cancer Res 2016; 35: 84.

37 Yusuf $R$, Frenkel K. Morphologic transformation of human breast epithelial cells MCF-10A: dependence on an oxidative microenvironment and estrogen/ epidermal growth factor receptors. Cancer Cell Int 2010; 10: 30.
38 Zhang Q, Stovall DB, Inoue K, Sui G. The oncogenic role of Yin Yang 1. Crit Rev Oncog 2011; 16: 163-197.

39 Bheda A, Creek KE, Pirisi L. Loss of p53 induces epidermal growth factor receptor promoter activity in normal human keratinocytes. Oncogene 2008; 27: 4315-4323.

40 Park WH. Anti-apoptotic effect of caspase inhibitors on $\left.\mathrm{H}_{2}\right) \mathrm{O}_{2}$ )-treated HeLa cells through early suppression of its oxidative stress. Oncol Rep 2014; 31: 2413-2421.

41 Bessette DC, Tilch E, Seidens T, Quinn MC, Wiegmans AP, Shi W et al. Using the MCF10A/MCF10CA1a breast cancer progression cell line model to investigate the effect of active, mutant forms of EGFR in breast cancer development and treatment using gefitinib. PLOS ONE 2015; 10: e0125232.

42 Nakai K, Hung MC, Yamaguchi H. A perspective on anti-EGFR therapies targeting triple-negative breast cancer. Am J Cancer Res 2016; 6: 1609-1623.

43 Fruehauf JP, Meyskens FL Jr. Reactive oxygen species: a breath of life or death? Clin Cancer Res 2007; 13: 789-794.

$44 \mathrm{Hu}$ Y, Rosen DG, Zhou Y, Feng L, Yang G, Liu J et al. Mitochondrial manganese-superoxide dismutase expression in ovarian cancer: role in cell proliferation and response to oxidative stress. J Biol Chem 2005; 280: 39485-39492.

45 Regelsberger G, Atzenhofer W, Ruker F, Peschek GA, Jakopitsch C, Paumann M et al. Biochemical characterization of a membrane-bound manganese-containing superoxide dismutase from the cyanobacterium Anabaena PCC 7120.J Biol Chem 2002; 277: 43615-43622.

46 Perekatt AO, Valdez MJ, Davila M, Hoffman A, Bonder EM, Gao N et al. YY1 is indispensable for Lgr5+ intestinal stem cell renewal. Proc Natl Acad Sci USA 2014; 111: 7695-7700.

47 El-Benna J, Dang PM, Gougerot-Pocidalo MA, Marie JC, Braut-Boucher F. p47phox the phagocyte NADPH oxidase/NOX2 organizer: structure, phosphorylation and implication in diseases. Exp Mol Med 2009; 41: 217-225.

48 Proskuryakov SY, Gabai VL. Mechanisms of tumor cell necrosis. Curr Pharm Des 2010; 16: 56-68.

49 Proskuryakov SY, Konoplyannikov AG, Gabai VL. Necrosis: a specific form of programmed cell death? Exp Cell Res 2003; 283: 1-16.

50 Vakkila J, Lotze MT. Inflammation and necrosis promote tumour growth. Nat Rev Immunol 2004; 4: 641-648.

51 Su Z, Yang Z, Xu Y, Chen Y, Yu Q. Apoptosis, autophagy, necroptosis, and cancer metastasis. Mol Cancer 2015; 14: 48.

52 Okayama H, Kohno T, Ishii Y, Shimada Y, Shiraishi K, Iwakawa R et al. Identification of genes upregulated in ALK-positive and EGFR/KRAS/ALK-negative lung adenocarcinomas. Cancer Res 2012; 72: 100-111.

53 Block Kl, Gyllenhaal C, Lowe L, Amedei A, Amin AR, Amin A et al. Designing a broad-spectrum integrative approach for cancer prevention and treatment. Semin Cancer Biol 2015; 35: S276-304.

54 Chen $X$, Song $M$, Zhang B, Zhang Y. Reactive oxygen species regulate $T$ cell immune response in the tumor microenvironment. Oxid Med Cell Longev 2016; 2016: 1580967

55 Arcucci A, Ruocco MR, Granato G, Sacco AM, Montagnani S. Cancer: an oxidative crosstalk between solid tumor cells and cancer associated fibroblasts. Bio Med Res Int 2016; 2016: 4502846.

56 Kageyama R, Merlino GT, Pastan I. Epidermal growth factor (EGF) receptor gene transcription. Requirement for Sp1 and an EGF receptor-specific factor. J Biol Chem 1988; 263: 6329-6336.

57 Golebiowski FM, Gorecki A, Bonarek P, Rapala-Kozik M, Kozik A Dziedzicka-Wasylewska M. An investigation of the affinities, specificity and kinetics involved in the interaction between the Yin Yang 1 transcription factor and DNA. FEBS J 2012; 279: 3147-3158.

58 Wang AM, Huang TT, Hsu KW, Huang KH, Fang WL, Yang MH et al. Yin Yang 1 is a target of microRNA-34 family and contributes to gastric carcinogenesis. Oncotarget 2014; 5: 5002-5016.

59 Chen QR, Yu LR, Tsang P, Wei JS, Song YK, Cheuk A et al. Systematic proteome analysis identifies transcription factor $\mathrm{YY} 1$ as a direct target of miR-34a. J Proteome Res 2011; 10: 479-487.

60 Misso G, Di Martino MT, De Rosa G, Farooqi AA, Lombardi A, Campani V et al. Mir-34: a new weapon against cancer? Mol Ther Nucleic Acids 2014; 3: e194.

61 Yin D, Ogawa S, Kawamata N, Leiter A, Ham M, Li D et al. miR-34a functions as a tumor suppressor modulating EGFR in glioblastoma multiforme. Oncogene 2013; 32: $1155-1163$.

62 Sundaram $\mathrm{P}$, Hultine $\mathrm{S}$, Smith LM, Dews M, Fox JL, Biyashev $\mathrm{D}$ et al. p53-responsive miR-194 inhibits thrombospondin-1 and promotes angiogenesis in colon cancers. Cancer Res 2011; 71: 7490-7501.

63 Sabharwal SS, Schumacker PT. Mitochondrial ROS in cancer: initiators, amplifiers or an Achilles' heel?. Nat Rev Cancer 2014; 14: 709-721.

64 Nicholson RI, Gee JM, Harper ME. EGFR and cancer prognosis. Eur J Cancer 2001; 37: S9-15. 
65 Zhang Y, He Q, Hu Z, Feng Y, Fan L, Tang Z et al. Long noncoding RNA LINP1 regulates repair of DNA double-strand breaks in triple-negative breast cancer. Nat Struct Mol Biol 2016; 23: 522-530.

66 Gazdar AF. Activating and resistance mutations of EGFR in non-small-cell lung cancer: role in clinical response to EGFR tyrosine kinase inhibitors. Oncogene 2009; 28: S24-S31.

67 Liccardi G, Hartley JA, Hochhauser D. EGFR nuclear translocation modulates DNA repair following cisplatin and ionizing radiation treatment. Cancer Res 2011; 71: 1103-1114.

68 Teng YH, Tan WJ, Thike AA, Cheok PY, Tse GM, Wong NS et al. Mutations in the epidermal growth factor receptor (EGFR) gene in triple negative breast cancer: possible implications for targeted therapy. Breast Cancer Res 2011; 13: R35.

69 Viale G, Rotmensz N, Maisonneuve P, Bottiglieri L, Montagna E, Luini A et al. Invasive ductal carcinoma of the breast with the 'triple-negative' phenotype: prognostic implications of EGFR immunoreactivity. Breast cancer research and treatment 2009; 116: 317-328.

70 Santarpia L, Qi Y, Stemke-Hale K, Wang B, Young EJ, Booser DJ et al. Mutation profiling identifies numerous rare drug targets and distinct mutation patterns in different clinical subtypes of breast cancers. Breast cancer research and treatment 2012; 134: 333-343.

71 Pani G, Galeotti T. Role of MnSOD and p66shc in mitochondrial response to p53. Antioxid Redox Signal 2011; 15: 1715-1727.
72 Yang $\mathrm{CH}$, Chou HC, Fu YN, Yeh CL, Cheng HW, Chang IC et al. EGFR overexpression in non-small cell lung cancers harboring EGFR mutations is associated with marked down-regulation of CD82. Biochimica et biophysica acta 2015; 1852: 1540-1549.

73 Cardoso SM, Rego AC, Penacho N, Oliveira CR. Apoptotic cell death induced by hydrogen peroxide in NT2 parental and mitochondrial DNA depleted cells. Neurochemistry international 2004; 45: 693-698.

74 Wang JY, Yang $\mathrm{CH}$, Yeh $\mathrm{CL}$, Lin $\mathrm{CH}$, Chen YR. NEAP causes down-regulation of EGFR, subsequently induces the suppression of NGF-induced differentiation in PC12 cells. J Neurochem 2008; 107: 1544-1555. Group. This work is licensed under a Creative Commons Attribution 4.0 International License. The images or other third party material in this article are included in the article's Creative Commons license, unless indicated otherwise in the credit line; if the material is not included under the Creative Commons license, users will need to obtain permission from the license holder to reproduce the material. To view a copy of this license, visit http://creativecommons.org/licenses/by/4.0/

(c) The Author(s) 2017

Supplementary Information accompanies this paper on the Oncogenesis website (http://www.nature.com/oncsis) 